\title{
Discrete-time Markovian arrival processes to model multi-state complex systems with loss of units and an indeterminate variable number of repairpersons
}

- Juan Eloy Ruiz-Castro, Mohammed Dawabsha, Francisco Javier Alonso

- Discrete-time Markovian arrival processes to model multi-state complex systems with loss of units and an indeterminate variable number of repairpersons

- Reliability Engineering and System Safety 174 (2018) 114-127

- DOI: https://doi.org/10.1016/j.ress.2018.02.019

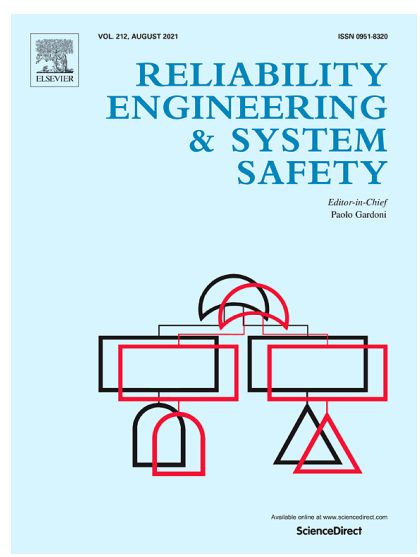




\title{
Discrete-time Markovian arrival processes to model multi-state complex systems with loss of units and an indeterminate variable number of repairpersons
}

\author{
Juan Eloy Ruiz-Castro*, Mohammed Dawabsha, Francisco Javier Alonso \\ Department of Statistics and Operational Research and IeMath Granada, Faculty of Science, University of Granada, Campus Fuentenueva s/n 18071, Spain
}

\section{A R T I C L E I N F O}

\section{Keywords:}

Multi-state system

Markovian arrival process

Preventive maintenance

Multiple and variable repairpersons

\begin{abstract}
A B S T R A C T
In this study, three discrete-time multi-state complex systems subject to multiple events are modeled, in a well structured form, as Markovian arrival processes with marked arrivals. The systems, ranked by the number of events affecting the online unit, have multiple and variable repairpersons, and the online unit are partitioned into performance stages. The first system is subject only to internal failures. The second, additionally, considers external shocks, which can produce any of three consequences; extreme failure, degradation of the internal performance of the online unit or cumulative damage. Failure may be repairable or non-repairable. The repair facility is composed of an indeterminate number of repairpersons. When a non-repairable failure occurs, the number of repairpersons may be modified. Finally, the third system includes preventive maintenance in combination with random inspections. Various measures are incorporated, in an algorithmic and computational form, in transient and stationary regimes. Costs and rewards are included in the model to optimize the system from different standpoints. The results of this study are implemented computationally with Matlab, and a numerical example shows the versatility of the modeling.
\end{abstract}

(C) 2018 Elsevier Ltd. All rights reserved.

\section{Introduction}

Serious damage and considerable financial losses are caused when a system failure occurs due to poor reliability. To avoid it, several reliability methodologies are commonly employed such as redundancy and maintenance policies. Redundant systems have been proposed in the reliability literature to solve different problems. The literature related to cold, warm and $k$-out-of- $n$ systems is extensive. For example, an optimization problem was addressed by Levitin et al. [12], who considered cold and warm standby groups. Wells [30] extended known analytic results to a case with repairable and non-repairable failures, while Levitin et al. [10] presented a method for evaluating the probability of mission success for an arbitrary redundancy level in several 1-out-of- $n$ subsystems where the environment is modeled by the Poisson process of shocks, by increasing the failure rate. Kim and Kim [9] suggested the exact reliability function for a cold standby redundant subsystem with an imperfect detector/switch. Recently, the reliability of a parallel system with active multicomponent and a single cold-standby unit has been investigated by Yongin et al. [34].

The provision of optimal maintenance is widely recommended as an effective way of minimizing system downtime and hence mainte-

\footnotetext{
* Corresponding author.

E-mail address: jeloy@ugr.es (J.E. Ruiz-Castro).
}

nance costs. Effective system maintenance improves overall reliability, prevents system failures and increases the benefit derived from the system. Several recent papers have made valuable contributions to improving the effectiveness of maintenance policies. Thus, Nakagawa [17] has discussed standard and advanced problems of maintenance policies for system reliability models, Qiu et al. [23] recently studied optimal maintenance policies for a competing-risk repairable system with a working state and a general number of failure modes undergoing periodic inspections, and Daneshkhah et al. [4] has developed probabilistic sensitivity analysis methods to study the sensitivity of optimized preventive maintenance decisions.

Nowadays, it is well known that the classical binary system of 'failure vs. operational state' has been extended by multi-state systems (MMS), a concept introduced in the mid-1970s by Murchland [16]. The efficiency of a system may vary according to the performance level of interest. MMS can have a finite number of performance/degradation stages. This approach has been studied using methods such as Markov and semiMarkov models, generating functions, Lz-transform and Monte Carlo simulations. Markov models have been considered to analyses the behavior of multi-state systems [13,22,31]. Yeh and Fiondella [33] determined the optimal redundancy allocation such that computer networks reliability is maximized. In this respect, too, Yi and Cui [32] have 
used Z-transform to analyze repairable aggregated semi-Markov ternary systems experiencing degradation and internal shocks, while Lisnianski et al. [14] proposed a method based on an Lz-transform of the discretestate continuous time Markov process, and also on Ushakov's Universal Generating Operator, to evaluate the sensitivity of an aging MSS under minimal repair. Finally, in this area, Levitin et al. [11] presented a novel Markov model of standby systems composed of multi-state elements in which, when an operating element fails, the standby element with the best technical state is chosen. The present paper describes an iterative algorithm to evaluate the reliability of the standby system.

Most studies of reliability focus on dynamic reliability systems in a continuous-time setting, while very few take into account the discretetime case. However, not all systems can be continuously monitored and some must be observed at certain times, for reasons such as the internal structure of the system or the need for periodic inspections. Thus, Warrington and Jones [29] proposed a method that integrates discrete event simulation with path sets to achieve a dynamic system. This method was applied to the analysis of Tornado aircraft movements. In the software reliability engineering literature, studies of the fault debugging environment have been made using discrete-time modeling. A discrete-time model suitable for a periodic debugging schedule, describing maximum likelihood estimation for the model parameters, was presented by Dewanji et al. [6]. Another discrete-time model of software reliability for such a scenario of periodic debugging has been developed by Das et al. [5]. Discrete-time nonhomogeneous Poisson process-based software reliability models must be developed and formulated taking into account the diversity of debugging scenarios. In this respect, Shatnawi [28] provides a new insight into the development of discrete-time modeling in software reliability engineering. Semi-Markov processes have also been considered to model discrete-time reliability systems $[2,8]$. Also, redundant Markovian multi-state systems have been studied in discrete time $[13,27]$. Therefore, reliability modelling in discrete time is necessary. In this respect, it is important to note that discrete time is not an immediate consequence of continuous time, and that relatively little research has focused on this question.

Different problems appear when complex reliability systems are modelled. The modelling process and the measures associated with the model have intractable expressions of highly complex applicability and interpretation. Furthermore, a reliability system can be subject to several types of events that can produce failure or degradation. Inter-event times are usually considered to be of a renewal type and to be independent and identically distributed. A solution for both these questions is to consider phase-type (PH) distributions and the Markovian Arrival Process (MAP). PH distributions were introduced and described in detail by Neuts $[19,20]$. Due to their valuable properties, many varieties of this class of distributions have been considered, in diverse branches of science and engineering, and applied in reliability studies. An interesting property highlighted by Neuts [20] is that any discrete distribution with finite support is a discrete $\mathrm{PH}$ distribution with a corresponding representation. These characteristics account for the widespread use of PH distributions in stochastic modelling.

On the other hand, the MAP is a well-structured counting process that enables reliability modelling to be developed in an algorithmic and computational form. This class of process, which is related to PH distributions, was introduced by Neuts [18] and comprehensively reviewed by Artalejo et al. [1] and He [7]. The MAP has attractive properties from the viewpoint of stochastic point processes. It is one of the most general classes of stochastic counting processes and contains many commonlyused arrival processes such as the Poisson process, the PH renewal process and the Markov-modulated Poisson process (MMPP). Moreover, the MAP is dense, meaning it can approximate an arbitrary stochastic point process to a given degree of accuracy. It has been applied in fields such as telecommunication and traffic queuing systems, reliability and industrial engineering. An extension of this class of process is the MAP with marked arrivals (MMAP). This approach is of interest in telecommunication, where different types of events are counted. In this respect, ap- proaches based on PH and the MAP has been extensively considered in reliability studies. Ruiz-Castro [25] modelled redundant complex MSS with different types of events, considering PH and MAPs, while Okamura et al. [21] addressed a parameter estimation problem of the MAP by proposing a numerical procedure for fitting a MAP and a MMPP in order to group data with an algorithm based on the expectation-maximization (EM) approach. Finally, Buchholz et al. [3] summarized the parameterization of PH distributions and MAPs, and analyzed different means of fitting a PH distribution and MMAP, considering the EM algorithm among others.

In reliability literature, complex systems are usually considered to be subject to non-repairable failures such that the failed unit is replaced by another, identical one. However, in many situations this assumption is not realistic and, indeed, may not even be necessary while the system is operational. Accordingly, Ruiz-Castro [24] and Ruiz-Castro and Fernández-Villodre [26] studied different standby complex systems with loss of units. The availability of multiple repairpersons is not usually assumed in reliability modelling and even less so is that of variable multiple repairpersons according to the number of units in the system.

This paper describes the algorithmic procedure used to model three multi-state complex cold standby systems with loss of units and an indeterminate and variable number of repairpersons, using MMAP. This paper makes two main contributions: on the one hand, we consider the loss of units with variable numbers of repairpersons; on the other, complex MMAPs are used to model complex systems with multiple events, after which the stationary distribution is determined. In the first of these systems, the online unit is only subject to failure by wear; the second extends this by including external shocks with diverse consequences, and the third includes inspections, so that the effects of preventive maintenance and of the variable number of repairpersons, depending on the number of units present in the system, are analyzed. An optimal maintenance policy enables policymakers to decide what level of degradation should be taken into account for preventive maintenance in response to an inspection, whether preventive maintenance is profitable and the optimum number of repairpersons at a given time. This study extends previous research in this area in the following ways: the online multistate unit passes through an indeterminate level of degradation, external shocks can produce several consequences (extreme failure, cumulative external damage, aggravation of the internal degradation or internal failure), preventive maintenance is performed in response to random inspections, the loss of units is considered (when a non-repairable failure occurs, the unit is not replaced while the system is operational), variable numbers of repairpersons are considered (the number of repairpersons depends on the number of units in the system), rewards and costs are included in the system and an optimizing example is shown and all results are expressed in algorithmic form, with PH distributions and Markovian arrival processes, with marked arrivals in discrete time (D-MMAP).

The applications considered range from performance and reliability/availability analyses of different configurations of non-repairable and repairable systems, to the development of maintenance strategies providing the desired system functioning, to the optimization of system structure, performance and maintenance schedules. In this respect, Markopoulos and Platis [15] considered MSS and semi-Markov modeling to restructure an IEEE 6 BUS RBTS energy system in order to enhance its reliability. Real-life systems are modeled in the present paper. The model presented can be applied in fields such as civil, industrial and computer engineering. For instance, in computer engineering, a computer server with three hard drives, two of which are available in cold standby, might be assumed. The online hard drive is periodically inspected by an installed monitoring program that analyses logic and physics parameters to detect possible errors. In civil engineering a fundamental element in well machinery is the drill bit. This is essential to advance the construction and it is subject to wear and/or breakage. Drill bits are very expensive and so they are regularly inspected and preventive maintenance is considered. New drill bits are kept in cold standby. 
The rest of this paper is organized as follows. The systems and the state-spaces are detailed in Section 2. In Section 3 the online unit and the repair facility are modeled. The MMAP for each system are developed in Section 4. Measures of the transient and the stationary distributions are obtained by considering matrix-analytics methods in Section 5, after which costs and rewards are introduced in Section 6. A numerical application illustrating the versatility of the model is presented in Section 7, and finally Section 8 presents the main conclusions drawn.

\section{The systems}

Three complex systems are described and modeled. The first is the most basic and the last, the most complex. The systems are available in cold standby, and the online unit is multi-state and subject to different types of events.

\section{SYSTEM I}

The online unit is multi-state and subject to internal repairable or non-repairable failure. The internal performance of the system is composed of several states which are partitioned into two well-differentiated groups: minor and major damage states, which reflect a low and high risk of failure, respectively.

SYSTEM II

The online unit is multi-state and subject to internal repairable or non-repairable failure and external shocks with different consequences, such as extreme failure of the online unit (non-repairable), degradation of the internal performance of the online unit, caused by a repairable internal failure, and cumulative external damage where if a threshold is reached a non-repairable failure occurs. Each time an external shock takes place, the cumulative external damage increases by passing through an external damage state. These cumulative external damage states are also well-differentiated in two groups: minor and major cumulative external damage states.

\section{SYSTEM III}

Random inspections are added to system II. If a major internal stage is reached and/or major external cumulative damage is observed by the inspection, the unit is sent to the repair facility for preventive maintenance. The time distributions for repairable failures and for preventive maintenance may be different.

Three main contributions are incorporated in these systems. The initial number of units in the system is general, $K$, each time that a unit undergoes a non-repairable failure is removed and the number of repairpersons in the repair facility is general and varies each time that a non-repairable failure occurs. The system continues working while there are units in the system. The number of repairpersons when there $k$ units in the system is denoted by $R_{k}$ where $1 \leq R_{k} \leq k$.

The systems are modeled and presented sequentially; the state-space, the modeling of the online unit, that of the repair facility, the associated MMAPs (from the online unit and the repair facility), the measures used and the costs produced. Examples are given in the modeling of the repair facility to illustrate the algorithmic approach used.

\subsection{Assumptions}

The cumulative assumptions for the systems are the following. SYSTEM I

Assumption 1. The internal operational time of the online unit is $\mathrm{PH}$ distributed with representation $(\alpha, T)$. The number of operational states is equal to $n$, and these are partitioned in minors (the first $n_{1}$ states) and majors states (states $n_{1}+1, \ldots, n$ ). The internal failure is repairable.

Assumption 2. When an internal failure occurs, it may be repairable or non-repairable. The probability of a repairable or non-repairable failure occurring depends on the transient states, and is given by the column vectors $\mathbf{T}_{r}^{0}$ and $\mathbf{T}_{n r}^{0}$, respectively. If the online unit is in the operational transient state $i$ then the probability of a repairable or non-repairable failure occurring is given by the $i$ th element of the column vectors $\mathbf{T}_{r}^{0}$ and $\mathbf{T}_{n r}^{0}$ respectively.

Assumption 3. When the online unit undergoes a non-repairable failure then it is removed and the number of the repairpersons is modified.

Assumption 4. The corrective repair time when the online unit fails is $\mathrm{PH}$ distributed with representation $\left(\boldsymbol{\beta}_{1}, \mathbf{S}_{1}\right)$. The order of this matrix is equal to $z_{1}$ (number of corrective repair phases).

Assumption 5. When the system is composed of only one unit and this one undergoes a non-repairable failure, the system is replaced by new and identical $K$-units system.

\section{SYSTEM II}

Assumption 6. External shocks over the online place occur according to a phase type renewal process. If the online place is busy, this event produces the failure of the unit. The time between two consecutive events is $\mathrm{PH}$ distributed with representation $(\gamma, \mathbf{L})$. The order of the matrix $\mathbf{L}$ is equal to $t$.

Assumption 7. One external shock can produce external cumulative damage, aggravation of the internal degradation where a repairable failure can produce or an extreme failure (non-repairable failure).

Assumption 8. External damage can pass through an indeterminate number of external degradation states. The number of external degradation states is equal to $d$, and these are partitioned in minors (the first $d_{1}$ states) and major states (states $d_{1}+1, \ldots, d$ ). If the external degradation state is $i$, then the external shock changes this one to state $j$ with probability $d_{i j}$. These probabilities are contained in the matrix $\mathbf{D}$. A cumulative external damage threshold is reached from the external damage states after an external shock through the probability column vector $\mathbf{D}^{0}$. If it occurs then the unit undergoes a non-repairable failure. Initially, previously to an external shock, the unit is in external degradation state 1 (no damage due to external shock). The initial distribution for external damage when one unit occupies the online place initially is $\omega=(1,0, \ldots, 0))_{1 x}$.

Assumption 9. One external shock can produce an extreme failure (non-repairable failure). It occurs with a probability equal to $\omega^{0}$.

Assumption 10. One external shock can produce modification in the internal degradation state. If the internal degradation state is $i$, then the external shock changes this one to state $j$ with probability $w_{i j}$. These probabilities are given in the matrix $\mathbf{W}$. An internal failure can occur due to this fact from any performance state with a probability column vector $\mathbf{W}^{0}$.

Fig. 1 shows a diagram for systems $I$ and $I I$. SYSTEM III

Assumption 11. While the online place is busy, random inspections can occur. The time between two consecutive inspections is $\mathrm{PH}$ distributed with representation $(\boldsymbol{\eta}, \mathbf{M})$. The order of the matrix $\mathbf{M}$ is equal to $\varepsilon$.

Assumption 12. When major internal or/and cumulative external damage is observed then the unit goes to preventive maintenance for preventive maintenance. Preventive maintenance time is $\mathrm{PH}$ distributed with representation $\left(\boldsymbol{\beta}_{2}, \mathbf{S}_{2}\right)$. The order of this matrix is equal to $z_{2}$ (number of preventive maintenance states).

Fig. 2 shows a diagram of system III.

\subsection{The state-space}

The state-space of the system is composed of macro-states. This statespace is different according to the systems.

SYSTEM I

The state space of the system is composed of two levels of macrostates. This state space is denoted by $S=\left\{\mathbf{U}^{K}, \mathbf{U}^{K-1}, \ldots, \mathbf{U}^{1}\right\}$, where $\mathbf{U}^{k}$ 


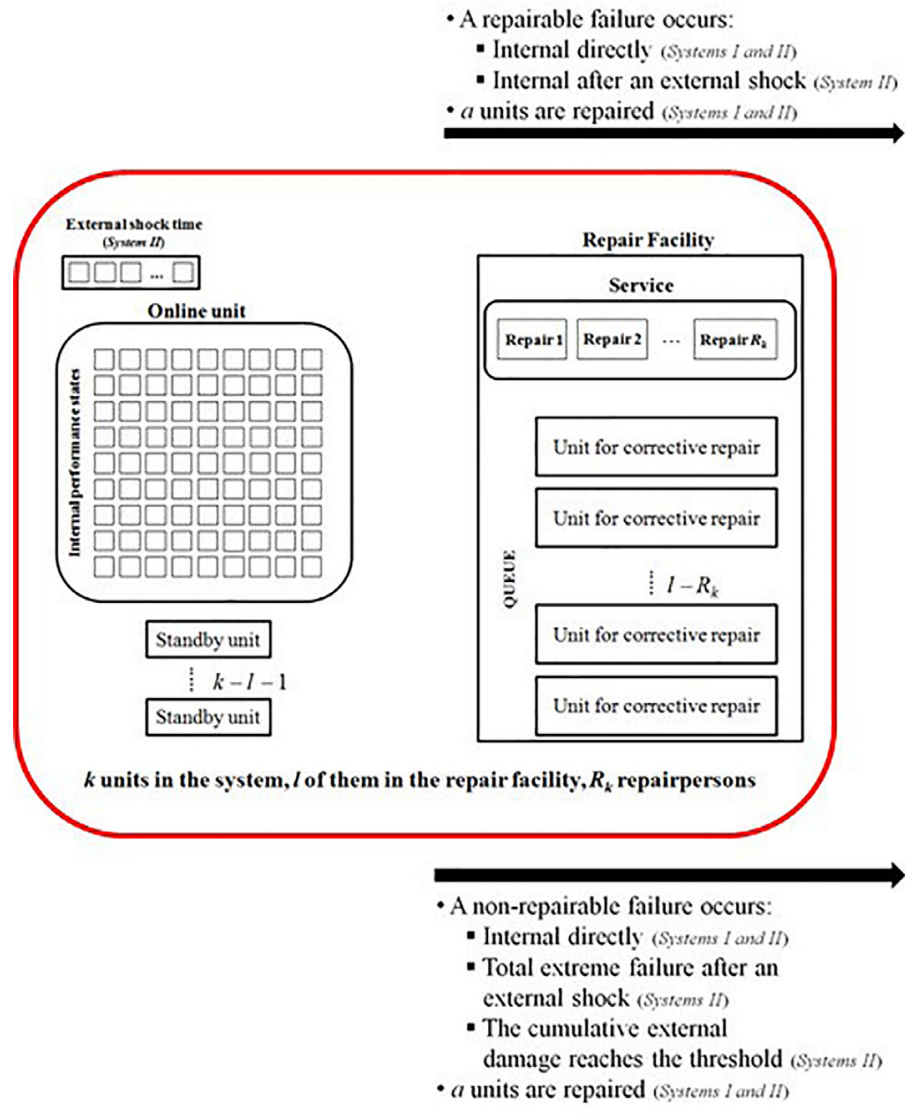

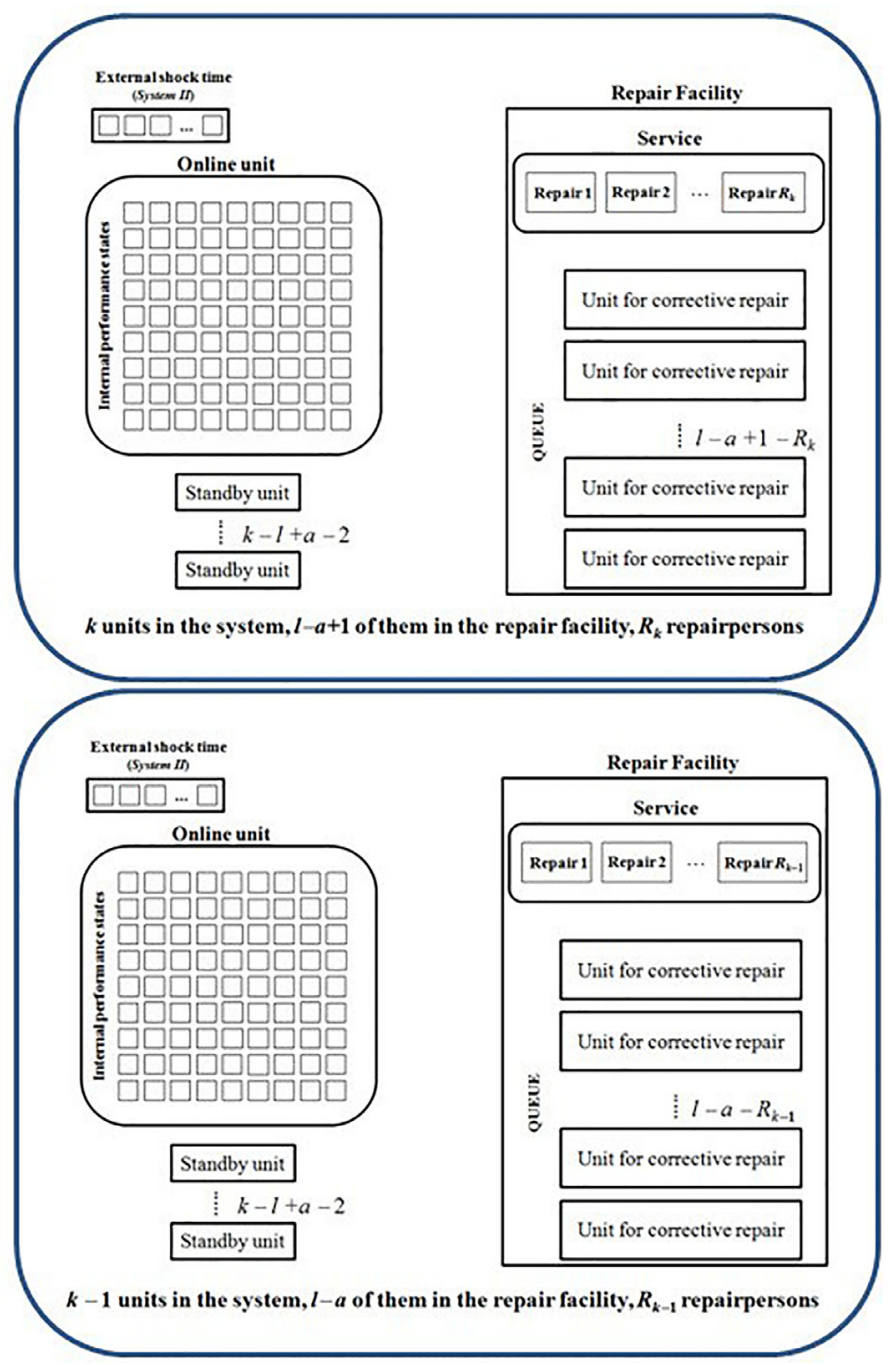

Fig. 1. Diagram of systems I and II. is the second level, containing the phases when there are $k$ units in the system. These macro-states are composed of the macro-states of the first level, $\mathbf{U}^{k}=\left\{\mathbf{E}_{0}^{k}, \mathbf{E}_{1}^{k}, \ldots, \mathbf{E}_{k}^{k}\right\}$ where $\mathbf{E}_{s}^{k}$ contains the phases when there are $k$ units in the system and $s$ units are in the repair facility. The phases of the system if the online unit is in state $i$ and the units in corrective repair, if any, are in states $r_{1}, \ldots, r_{\min \left\{s, R_{k}\right\}}$ are for $k=1, \ldots, K$ and $s=1, \ldots$, $k-1$,

$\mathbf{E}_{0}^{k}=\{(k ; i) ; i=1, \ldots, n\} ; \mathbf{E}_{s}^{k}=\left\{\left(k, s ; i, r_{1}, \ldots, r_{\min }\left\{s, R_{k}\right\}\right) ; i=1, \ldots, n\right.$,

$\left.\mathrm{r}_{h}=1, \ldots, z_{1}, h=1, \ldots, \min \left\{s, R_{k}\right\}\right\}$

$\mathbf{E}_{k}^{k}=\left\{\left(k, k ; r_{1}, \ldots, r_{\min }\left\{s, R_{k}\right\}\right) ; \mathrm{r}_{h}=1, \ldots, z_{1}, h=1, \ldots, \min \left\{s, R_{k}\right\}\right\}$

\section{SYSTEM II}

The state-space of system II is again composed of two levels, but in this case the states of the inspection time, $j$, and the external cumulative damage, $u$, are included. Then, for $k=1, \ldots, K$ and $s=1, \ldots, k-1$,

$\mathbf{E}_{0}^{k}=\{(k, 0 ; i, j, u) ; i=1, \ldots, n, j=1, \ldots, t, u=1, \ldots, d\} ;$

$\mathbf{E}_{k}^{k}=\left\{\left(k, s ; j, r_{1}, \ldots, r_{\min \left\{s, R_{k}\right\}}\right) ; j=1, \ldots, t, \mathrm{r}_{h}=1, \ldots, z_{1}\right.$,

$\left.h=1, \ldots, \min \left\{s, R_{k}\right\}\right\}$

$\mathbf{E}_{s}^{k}=\left\{\left(k, s ; i, j, u, r_{1}, \ldots, r_{\min \left\{s, R_{k}\right\}}\right) ; i=1, \ldots, n, j=1, \ldots, t, u=1, \ldots, d\right.$,

$\left.\mathrm{r}_{h}=1, \ldots, z_{1}, h=1, \ldots, \min \left\{s, R_{k}\right\}\right\}$

\section{SYSTEM III}

The state space of System III is composed of three levels of macrostates. In this case the order of the units in the repair facility has to be saved in memory, as there are two types of repair, corrective and preventive maintenance. For this reason, the macro-state $\mathbf{E}_{s}^{k}$ is composed of the first level of macro-states $\mathbf{E}_{i_{1}, \ldots, i_{s}}^{k}$. These macro-states contain the phases when there are $k$ units in the system, with $s$ of them in the repair facility, and the type of repair is given by the ordered sequence $i_{1}, \ldots, i_{s}$. The values of $i_{l}$ are equal to 1 or 2 if the unit is in corrective repair or preventive maintenance, respectively. Then, for $k=1, \ldots, K$,

$\mathbf{E}_{0}^{k}=\{(k, 0 ; i, j, u, m) ; i=1, \ldots, n, j=1, \ldots, t, u=1, \ldots, d, m=1, \ldots, \varepsilon\}$ $\mathbf{E}_{s}^{k}=\left\{\mathbf{E}_{i_{1}, \ldots, i_{s}}^{k} ; i_{l}=1,2 ; l=1, \ldots, s, j=1, \ldots, s\right\}$

for $s=1, \ldots, k$ where

$\mathbf{E}_{i_{1}, \ldots, i_{s}}^{k}=\left\{\left(k, s ; i, j, u, m, r_{1}, \ldots, r_{\min \left\{s, R_{k}\right\}}\right) ; i=1, \ldots, n, j=1, \ldots, t\right.$, $\left.u=1, \ldots, d, m=1, \ldots, \varepsilon, \mathrm{r}_{h}=1, \ldots, z_{i_{h}}, h=1, \ldots, \min \left\{s, R_{k}\right\}\right\}$

for $s=1, \ldots, k-1$ and

$\mathbf{E}_{i_{1}, \ldots, i_{k}}^{k}=\left\{\left(k, s ; j, r_{1}, \ldots, r_{R_{k}}\right) ; j=1, \ldots, t, r_{h}=1, \ldots, z_{i_{h}}, h=1, \ldots, R_{k}\right\}$.

The phase $\left(k, s ; i, j, u, m, r_{1}, \ldots, r_{\min \left\{s, R_{k}\right\}}\right)$ indicates that there are $k$ units in the system of which $s$ of them are in the repair facility, the internal performance is in state $i$, the external shock time is in state $j$, the cumulative damage undergone by external shocks is given by $u, m$ is the 


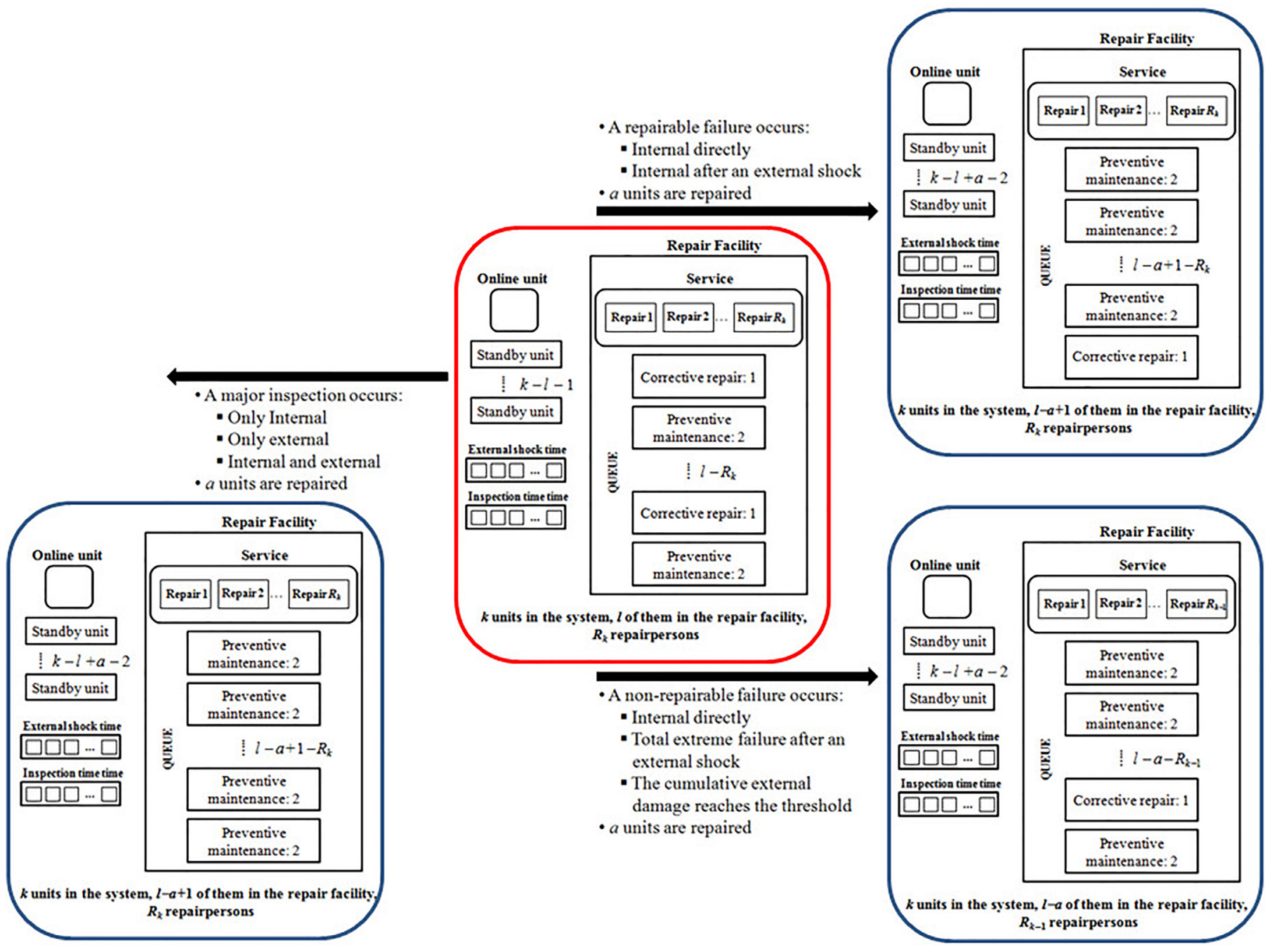

Fig. 2. Diagram of system III.

phase of the inspection time and $r$ is the corrective repair/preventive maintenance phase for the units that are being repaired in the repair facility.

\section{Modeling the systems}

The systems are governed by a Markov process vector in discrete time with the state space described in Section 2.2. To model any proposed complex system, the behavior of the online unit and of the repair facility must be described separately. This section shows the case of System III but analogous reasoning can be performed for Systems I and II. The corresponding matrices for all systems are given in Appendices $A$ and $B$.

\subsection{Modeling the online unit}

The online unit of system III can undergo different types of events. These ones are partitioned as:

$A_{1}$ : Internal repairable failure due to internal degradation

$A_{2}$ : Internal repairable failure due to external shocks

$B_{1}$ : Major revision for only major internal degradation after inspection

$B_{2}$ : Major revision for only major external cumulative damage after inspection

$B_{3}$ : Major revision in both cases (internal and external cumulative damage)

$C_{1}$ : Non-repairable failure due to internal degradation
$\mathrm{C}_{2}$ : Non-repairable failure due to one external shock $O$ : No events

The transition for each event affecting the online unit is obtained as follows. The repairable case $\left(A_{1}, A_{2}\right)$ is discussed below, and the remaining cases are shown in Appendix $A$. Throughout the paper, the column vector e denotes a column vector of ones with appropriate order, $\mathbf{e}_{a}$ a column vector of 1 's with order $a$, $\mathbf{I}$ is the identity matrix with appropriate order, $I_{\{\text {condition }\}}$ is the indicatory function that it is equal to 1 if the condition is true and 0 otherwise and $\otimes$ is the Kronecker product. An internal repairable failure $\left(A_{1}\right)$ can occur due to internal degradation or after an external shock. In the first case, the online unit undergoes an internal repairable failure and another unit occupies the online place $\left(\mathbf{T}_{r}^{0} \boldsymbol{\alpha}\right)$; an external shock occurs or does not $\left(\mathbf{L}^{0} \gamma, \mathbf{L}\right.$ respectively); if it does, cumulative damage occurs but there is no non-repairable failure, Dew $\left(1-\omega^{0}\right)$. If an inspection takes place at the same time, the unit undergoes a repairable failure and the inspection time begins for the new online unit $\left(\mathbf{e}_{\varepsilon} \eta\right)$. This transitions is governed by

$\mathbf{H}_{\text {rep }}^{1}=\left[\mathbf{T}_{r}^{0} \boldsymbol{\alpha} \otimes \mathbf{L} \otimes \mathbf{e} \boldsymbol{\omega}+\mathbf{T}_{r}^{0} \boldsymbol{\alpha} \otimes \mathbf{L}^{0} \boldsymbol{\gamma} \otimes \mathbf{D e} \boldsymbol{\omega}\left(1-\omega^{0}\right)\right] \otimes \mathbf{e}_{\varepsilon} \eta$.

If the online unit is the only operational unit and a repair does not occur, then none unit will occupy the online place at the next time. In this case,

$\mathbf{H}_{\text {rep }}^{\prime 1}=\left[\mathbf{T}_{r}^{0} \otimes \mathbf{L} \otimes \mathbf{e}_{d}+\mathbf{T}_{r}^{0} \otimes \mathbf{L}^{0} \boldsymbol{\gamma} \otimes \mathbf{D e}\left(1-\omega^{0}\right)\right] \otimes \mathbf{e}_{\varepsilon}$.

A similar reasoning can be applied when an external shock provokes an internal repairable failure $\left(A_{2}\right)$. In this case, an external shock oc- 
curs $\left(\mathbf{L}^{0} \gamma\right)$ and the internal behavior is modified to address the internal failure $\left(\mathbf{T W}^{0} \boldsymbol{\alpha}\right)$. This shock does not provoke a non-repairable failure (Dew $\left.\left(1-\omega^{0}\right)\right)$. The transition matrix is governed by

$\mathbf{H}_{\mathrm{rep}}^{2}=\left[\mathbf{T} \mathbf{W}^{0} \boldsymbol{\alpha} \otimes \mathbf{L}^{0} \boldsymbol{\gamma} \otimes \mathbf{D e \omega}\left(1-\omega^{0}\right)\right] \otimes \mathbf{e}_{\varepsilon} \eta$.

Analogously, if at the next time the online place is empty then

$\mathbf{H}_{\text {rep }}^{\prime 2}=\left[\mathbf{T W}^{0} \otimes \mathbf{L}^{0} \boldsymbol{\gamma} \otimes \mathbf{D e}\left(1-\omega^{0}\right)\right] \otimes \mathbf{e}_{\varepsilon}$.

The rest of the matrices are given in Appendix $A$.

\subsection{Modeling the repair facility}

As mentioned above, the modeling is developed for System III, but the method described is valid for Systems I and II if only corrective repair and non-repairable failures are considered. The transition matrix for the repair facility depends on the number of repairpersons, the number of units in the repair facility, the number of units that are successfully repaired and the type of failure (if any) of the online unit. The number of repairpersons when there are $\mathrm{k}$ units in the system, with $k \leq K$, is given by $R_{k} \leq k$ and the number of units in the repair facility is denoted as $l$. Let $a$ be the number of units which finish the repair. Let $k_{h}$ be the ordinal of the repairpersons who concluded the repair, and let $i_{h}$ and $j_{h}$ be the type of repair (corrective, 1 , preventive maintenance, 2 ) for the ordered units, after and before the transition, respectively. The online unit can undergo two types of events that can require the unit to be sent to the repair facility: repairable failure or major inspection. This fact is included in the modeling through the variable $m r$, which is equal to 0 if the unit does not undergo an event and 1 if a repairable failure or a major inspection occurs. The online unit is also subject to non-repairable failure. If this occurs, it is denoted by $n r=1$, otherwise it is equal to 0 .When a non-repairable failure occurs, the number of repairpersons can be modified. In this case, if there are fewer repairpersons after a transition than remaining units being repaired, some of these units will be returned to the queue in the repair facility. The number of units to be returned is denoted by $b$. This value is given by

$b=\max \left\{\min \left\{l, R_{k}\right\}-a-I_{\{n r=1\}} R_{k-1}-I_{\{n r=0\}} R_{k}, 0\right\}$.

To model the behavior of the repair facility we define the following matrix function that governs the behavior in one transition of the units that are being repaired where the order of the units repaired are specified. This function is given by

$$
\begin{aligned}
& C\left(k, l, a, b ; k_{1}, \ldots, k_{a} ; i_{1}, \ldots, i_{l-a+m r} ; j_{1}, \ldots, j_{l}\right) \\
& = \begin{cases}\mathbf{S}(1) \otimes \cdots \otimes \mathbf{S}\left(\min \left\{l, R_{k}\right\}\right) ; & i \sum_{s-\sum_{z=1}^{a} I_{\left\{k_{z}<s\right\}}} \text { otherwise } \\
\mathbf{0} ; & =j_{s} ; s=1, \ldots, l ; s \neq k_{z}, \forall z .\end{cases}
\end{aligned}
$$

for $k \leq K, l \geq 1, a \geq 1, b \geq 0$, where

$$
S(h)=\left\{\begin{array}{lll}
\mathbf{S}_{j_{h}}^{0} & ; \quad \exists z \in\{1, \ldots, a\} \mid h=k_{z} \\
\mathbf{e}-\mathbf{S}_{j_{h}}^{0} & ; \quad \begin{array}{l}
h \text { is the ordinal of the last } b \text { units being repaired } \\
\text { without ending }
\end{array} \\
\mathbf{S}_{j_{h}} & ; \quad \text { otherwise }
\end{array}\right.
$$

If $a=0$, then the definition is analogous but we will consider the following notation

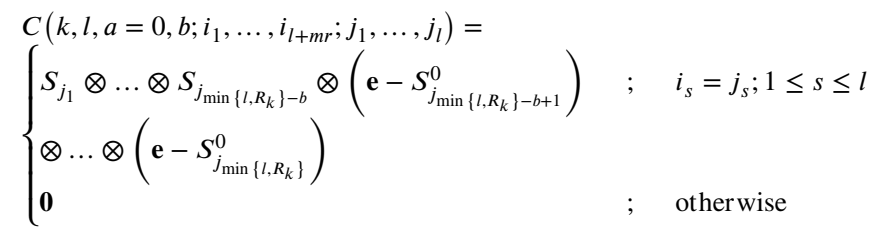

If $a=\min \left\{l, R_{k}\right\}$, then the definition is analogous but we will consider the following notation

$$
C\left(k, l, a=\min \left\{l, R_{k}\right\}, 0 ; j_{1}, \ldots, j_{l}\right)=S_{j_{1}}^{0} \otimes \ldots \otimes S_{j_{\min }\left\{l, R_{k}\right\}}^{0} .
$$

Example 1. For instance, we assume a system composed of 4 repairpersons and 6 units $\left(k=6, R_{6}=4\right), 5$ of them in the repair facility ( $l=5$; preventive maintenance, corrective repair, preventive maintenance, corrective repair and preventive maintenance respectively). At the next time three units that are being repaired finishes the repair, and the online unit undergoes a non repairable failure. The number of repairpersons is only two when the system is composed of 5 units $\left(R_{5}=2\right)$ and the units in the repair facility after non-repairable failure are types corrective repair and preventive maintenance respectively.

In this case the number of units that are devolved to the queue in the repair facility is $b=\max \{\min \{5,4\}-3-2,0\}=0$. If the first three units are repaired then this transition for the units that was being repaired with the established order is

$$
\begin{aligned}
& C\left(k=6, l=5, a=3, b=0 ; k_{1}=1, k_{2}=2, k_{3}=3 ; i_{1}=1, i_{2}=2 ; j_{1}=2, j_{2}\right. \\
& \left.\quad=1, j_{3}=2, j_{4}=1, j_{5}=2\right)=S_{2}^{0} \otimes S_{1}^{0} \otimes S_{2}^{0} \otimes S_{1} .
\end{aligned}
$$

From this matrix function the transition probability, if only $a$ is known and the order is not specified, is given by

$$
\begin{aligned}
& B\left(k, l, a, b ; i_{1}, \ldots, i_{l-a+m r} ; j_{1}, \ldots, j_{l}\right) \\
& =\left\{\begin{array}{lll}
C\left(k, l, 0, b ; i_{1}, \ldots, i_{l+m r}, j_{1}, \ldots, j_{l}\right) & ; & a=0, b \geq 0, l>0 \\
\min \left\{l, R_{k}\right\}-a+1 \min \left\{l, R_{k}\right\}-a+2 & \min \left\{l, R_{k}\right\} & \\
\sum_{k_{1}=1} \sum_{k_{2}=k_{1}+1} \ldots \sum_{k_{a}=k_{a-1}+1} & ; & a>0, a \neq \min \left\{l, R_{k}\right\} \\
C\left(k, l, a, b ; k_{1}, \ldots, k_{a} ; i_{1}, \ldots, i_{l-a+m r}, j_{1}, \ldots, j_{l}\right) & ; & a=\min \left\{l, R_{k}\right\}, \\
C\left(k, l, a, 0 ; j_{1}, \ldots, j_{l}\right) & ; & a=0, b=0, l=0
\end{array}\right.
\end{aligned}
$$

Example 2. If the Example 1 is considered, then transition probability matrix when the order of the units repaired are not specified is given by

$$
\begin{aligned}
& B\left(k=6, l=5, a=3, b=0 ; i_{1}=1, i_{2}=2 ; j_{1}=2, j_{2}=1, j_{3}=2, j_{4}\right. \\
& \left.\quad=1, j_{5}=2\right)=S_{2}^{0} \otimes S_{1}^{0} \otimes S_{2}^{0} \otimes S_{1}+\mathbf{0}+S_{2}^{0} \otimes S_{1} \otimes S_{2}^{0} \otimes S_{1}^{0}+\mathbf{0} .
\end{aligned}
$$

After one transition, new units that were in queue or not can entry in repair. The number of units that will begin the repair at the next time is given by

$$
\begin{aligned}
\varepsilon= & \min \left\{\max \left\{0, l-R_{k}\right\}+m r, I_{\{n r=1\}} R_{k-1}+I_{\{n r=0\}} R_{k}\right. \\
& \left.-\min \left\{\min \left\{R_{k}, l\right\}-a, I_{\{n r=1\}} R_{k-1}+I_{\{n r=0\}} R_{k}\right\}\right\} .
\end{aligned}
$$

The matrix function that governs the transition probability of the repair facility when $a$ of $l$ units are repaired for $l>0$ and $a \neq l$ is given by

$$
\begin{aligned}
& E\left(k, l, a, b ; i_{1}, \ldots, i_{l-a+m r} ; j_{1}, \ldots, j_{l} ; m r, n r\right) \\
& \quad=\left\{\begin{array}{lll}
B\left(k, l, a, 0 ; i_{1}, \ldots, i_{l-a+m r} ; j_{1}, \ldots, j_{l}\right) \otimes & ; & \varepsilon>0 \\
\boldsymbol{\beta}^{i_{\min }\left(l, R_{k}\right)-a+1} \otimes \ldots \otimes \boldsymbol{\beta}^{i_{\min }\left(l, R_{k}\right)-a+\varepsilon} & \\
B\left(k, l, a, b ; i_{1}, \ldots, i_{l-a+m r} ; j_{1}, \ldots, j_{l}\right) & ; & \varepsilon=0 \\
\mathbf{0} & ; & \text { otherwise }
\end{array}\right.
\end{aligned}
$$

If $l=0$ or $a=l$ with $l \leq R_{k}$ then this function is denoted as

$$
\begin{aligned}
& E(k, l=0, a=0, b=0 ; m r=0, n r=0,1)=1, \\
& E\left(k, l=0, a=0, b=0 ; i_{m r} ; m r=1, n r=0\right)=\boldsymbol{\beta}^{i_{m r}}, \\
& E\left(k, l, a=l, b=0 ; j_{1}, \ldots, j_{l} ; m r=0, n r=0,1\right)=B\left(k, l, l, 0 ; j_{1}, \ldots, j_{l}\right), \\
& E\left(k, l, a=l, b=0 ; i_{m r} ; j_{1}, \ldots, j_{l} ; m r=1, n r=0\right) \\
& \quad=B\left(k, l, l, 0 ; j_{1}, \ldots, j_{l}\right) \otimes \boldsymbol{\beta}^{i_{m r}}
\end{aligned}
$$

Example 3. If the Examples 1 and 2 are considered, then the number of units that entry in repair is given by $\varepsilon=$ $\min \{\max \{0,1\}+0,2-\min \{4-3,2\}\}=1$. Therefore, the transition probability for the repair facility is given by

$$
\begin{aligned}
& E\left(k=6, l=5, a=3, b=0 ; i_{1}=1, i_{2}=2 ; j_{1}=2, j_{2}=1, j_{3}=2, j_{4}=1,\right. \\
& \left.\quad j_{5}=2 ; m r=0, n r=1\right) \\
& \quad=\left(S_{2}^{0} \otimes S_{1}^{0} \otimes S_{2}^{0} \otimes S_{1}+S_{2}^{0} \otimes S_{1} \otimes S_{2}^{0} \otimes S_{1}^{0}\right) \otimes \boldsymbol{\beta}^{i_{2}=2}
\end{aligned}
$$




\section{The Markovian arrival processes with marked arrivals}

The systems I, II and III, are modeled by different MMAPs by considering the different types of events described in Section 3.1. The MMAPs for the different systems have the following representations,

Model I: $\left(\mathbf{D}^{O}, \mathbf{D}^{A_{1}}, \mathbf{D}^{C_{1}}, \mathbf{D}^{F C_{1}}\right)$

Model II: $\left(\mathbf{D}^{O}, \mathbf{D}^{A_{1}}, \mathbf{D}^{A_{2}}, \mathbf{D}^{C_{1}}, \mathbf{D}^{C_{2}}, \mathbf{D}^{F C_{1}}, \mathbf{D}^{F C_{2}}\right)$

Model III: ( $\left.\mathbf{D}^{O}, \mathbf{D}^{A_{1}}, \mathbf{D}^{A_{2}}, \mathbf{D}^{B_{1}}, \mathbf{D}^{B_{2}}, \mathbf{D}^{B_{3}}, \mathbf{D}^{C_{1}}, \mathbf{D}^{C_{2}}, \mathbf{D}^{F C_{1}}, \mathbf{D}^{F C_{2}}\right)$.

$F C_{1}$ and $F C_{2}$ denote the same that $C_{1}$ and $C_{2}$ respectively when only one unit is present in the system. These events will be used to count the number of new systems by time.

The matrix $\mathbf{D}^{Y}$ contains the transition probabilities when the event $Y$ has occurred for $Y=O, A_{1}, A_{2}, B_{1}, B_{2}, B_{3}, C_{1}, C_{2}, F C_{1}, F C_{2}$.

The matrix $\mathbf{D}^{Y}$ is built following three matrix block levels, always when the event $Y$ occurs. The third level corresponds to the transitions from the macro-state $\mathbf{U}^{k}$ to $\mathbf{U}^{k}$ or $\mathbf{U}^{k-1}$. These matrix blocks are composed of the matrices $\mathbf{D}_{l h}^{Y, k}$ which correspond to the transitions between the macro-states from $\mathbf{E}_{l}^{k}$ to either $\mathbf{E}_{h}^{k}$ or $\mathbf{E}_{h}^{k-1}$ (level 2).

Finally, when preventive maintenance is introduced (system III) several types of repairing can be produced. Therefore, the type of failure of the units in the repair facility has to be saved in memory. The matrices $\mathbf{D}_{l h}^{Y, k}$ are composed of matrix blocks corresponding to the transition from the macro-states $\mathbf{E}_{i_{1}, \ldots, i_{l}}^{k}$ to $\mathbf{E}_{i_{1}, \ldots, i_{h}}^{k}$ or $\mathbf{E}_{i_{1}, \ldots, i_{h}}^{k-1}$. The matrix block $\mathbf{D}_{l h}^{Y, k}\left(i_{1}, \ldots, i_{h} ; j_{1}, \ldots, j_{l}\right)$ contains the transition probabilities described above where the type of repair in the repair facility is ordered for the case before and after transition. These blocks are built by considering the matrices $\mathbf{H}$ defined in Section 3.1 and developed in Appendix A (level 1). Next, the case $A_{1}$ for the model III, a repairable internal failure occurs, is described in detail. The rest is given in an algorithmic form in Appendix $B$.

Building the matrix $\mathbf{D}^{A_{1}}$

This matrix $\mathbf{D}^{A_{1}}$ is a matrix block that governs the transitions when an internal repairable failure occurs. Then this matrix is a diagonal matrix block $\mathbf{D}^{A_{1}}=\operatorname{diag}\left(\mathbf{D}^{A_{1}, K}, \mathbf{D}^{A_{1}, K-1}, \mathbf{D}^{A_{1}, K-2}, \ldots, \mathbf{D}^{A_{1}, 1}\right)$ given that a non-repairable failure does not occur. The matrix block $\mathbf{D}^{A_{i}, k}$ contains the transitions when this fact occurs with $k$ units in the system. The elements of the matrix $\mathbf{D}^{A_{1}, k}$ for $k=1, \ldots, K$ are matrix blocks by considering the number of units in the system, $l$ and $h$ before and after the transition respectively. It is given by

$\mathbf{D}^{A_{1}, k}=\left(\mathbf{D}_{l h}^{A_{1}, k}\right)_{l, h=0, \ldots, k}$

where $\mathbf{D}_{l h}^{A_{1}, k}=\mathbf{0}$ if $h>l+1$ or $h<l+1-\min \{l, R(k)\}$ or $l=k$.

Finally, these matrices are again composed of matrix blocks by taking into account the order of the units in the repair facility. Thus, if there are $k$ units in the system, $l$ of them in the repair facility with ordered type of failure $\left(j_{1}, \ldots, j_{l}\right), a$ units are repaired and one repairable failure occurs then the transition matrix is given by

$\mathbf{D}_{l, l+1-a}^{A_{1}, k}\left(i_{1}, \ldots, i_{l+1-a-1}, 1 ; j_{1}, \ldots, j_{l}\right)=\left(\mathbf{H}_{\mathrm{rep}}^{1} \boldsymbol{I}_{\{l<k-1 \operatorname{lor} a>0\}}+\mathbf{H}_{\mathrm{rep}}^{\prime 1} \boldsymbol{I}_{\{l=k-1 \mathrm{and} a=0\}}\right)$

$\otimes E\left(k, l, a, 0 ; i_{1}, \ldots, i_{l+1-a-1}, 1 ; j_{1}, \ldots, j_{l} ; 1,0\right)$,

for $l=1, \ldots, k-1 ; a=0, \ldots, \min \left\{l, R_{k}\right\}$ and $k>1$.

The matrix is obtained according to the following algorithm.

1. $\mathbf{D}^{A_{1}}=\operatorname{diag}\left(\mathbf{D}^{A_{1}, K}, \mathbf{D}^{A_{1}, K-1}, \mathbf{D}^{A_{1}, K-2}, \ldots, \mathbf{D}^{A_{1}, 1}\right)$

2. $\mathbf{D}^{A_{1}, k}=\left(\mathbf{D}_{l h}^{A_{1}, k}\right)_{l, h=0, \ldots, k}$ for $k=1, \ldots, K$

3. Building blocks $\mathbf{D}_{l h}^{A_{1}, k}$ for $l+1-\min \{l, R(k)\} \leq h \leq l+1$ and $l \neq k$ 3.1 If $l=0$

a. Calculating $\quad \mathbf{D}_{01}^{A_{1}, k}(1)=\left(\mathbf{H}_{\text {rep }}^{1} I_{\{k>1\}}+\mathbf{H}_{\text {rep }}^{\prime 1} I_{\{k=1\}}\right) \otimes$ $E(k, 0,0,0 ; 1 ; 1,0)$

a.1. If $k>1 \rightarrow \mathbf{H}_{\text {rep }}^{1}$ else $\mathbf{H}_{\text {rep }}^{\prime 1}$

a.2. Function $E(k, 0,0,0 ; 1 ; 1,0)=B(k, 0,0,0 ; 1) \otimes \beta^{1}$

a.2.1. Calculating $\varepsilon$ and $b$ (in this case it is equal to one and zero respectively)

a.2.2. Calculating function $B(k, 0,0,0 ; 1)=1$
3.2. For $l=1, \ldots, k-1 ; a=0, \ldots, \min \left\{l, R_{k}\right\}$ with $k>1$

a. Calculating

$\mathbf{D}_{l, l+1-a}^{A_{1}, k}\left(i_{1}, \ldots, i_{l+1-a-1}, 1 ; j_{1}, \ldots, j_{l}\right)=\left(\mathbf{H}_{\text {rep }}^{1} I_{\{l<k-1 \text { ora } a 0\}}+\mathbf{H}_{\text {rep }}^{\prime 1} I_{\{l=k-1 \text { and } a=0\}}\right)$

$\otimes E\left(k, l, a, 0 ; i_{1}, \ldots, i_{l+1-a-1}, 1 ; j_{1}, \ldots, j_{l} ; 1,0\right)$

a.1. If $l<k-1$ or $a>0 \rightarrow \mathbf{H}_{\mathrm{rep}}^{1}$ else $\mathbf{H}_{\mathrm{rep}}^{\prime 1}$

a.2. Function $E\left(k, l, a, 0 ; i_{1}, \ldots, i_{l+1-a-1}, 1 ; j_{1}, \ldots, j_{l} ; 1,0\right)$

a.2.1. Calculating $\varepsilon$ and $b$ (in this case $b$ is equal to zero)

a.2.2. Calculating function

$$
B\left(k, l, a, 0 ; i_{1}, \ldots, i_{l-a+1-1}, 1 ; j_{1}, \ldots, j_{l}\right)
$$

The rest of matrices are given in Appendix $B$.

\section{The transient and stationary distribution and measures}

The transient and stationary distributions have been built so as several measures of interest. These measures are developed for the system III. The other models can be achieved in a similar way.

\subsection{The transient distribution}

Once built the D-MMAP, the transition probability matrix that governs the discrete Markov chain associated to the system III is given by $\mathbf{D}=\mathbf{D}^{0}+\mathbf{D}^{A_{1}}+\mathbf{D}^{A_{2}}+\mathbf{D}^{B_{1}}+\mathbf{D}^{B_{2}}+\mathbf{D}^{B_{3}}+\mathbf{D}^{C_{1}}+\mathbf{D}^{C_{2}}+\mathbf{D}^{F C_{1}}+\mathbf{D}^{F C_{2}}$. Given the initial distribution of the system $\theta$, the transient distribution is worked out as $\mathbf{p}^{v}=\boldsymbol{\theta} \mathbf{D}^{v}$. Therefore, the probability of being in the macro-state $\mathbf{E}_{s}^{k}$ at time $v$ is the corresponding part of $\mathbf{p}^{v}$ and it is denoted by $\mathbf{p}_{\mathbf{E}_{s}^{k}}^{v}$.

\subsection{Stationary distribution}

The stationary distribution, $\pi$, has been built solving the balance equations by applying matrix analytic methods. It is well known that the stationary distribution verifies $\boldsymbol{\pi} \mathbf{D}=\boldsymbol{\pi}$ and $\pi \mathbf{e}=1$. This system has been solved for the macro-state $\mathbf{E}^{k}, k$ units in the system. The stationary distribution for this macro-state is denoted by $\pi_{\mathrm{E}^{k}}$. Then, the stationary distribution is $\pi=\left(\boldsymbol{\pi}_{\mathbf{E}^{K}}, \boldsymbol{\pi}_{\mathbf{E}^{K-1}}, \ldots, \boldsymbol{\pi}_{\mathbf{E}^{1}}\right)$. From the MMAP the transition probability matrices for the transition from $\mathbf{E}^{k}$ to $\mathbf{E}^{k}$ or $\mathbf{E}^{k-1}$ are denoted by

$\mathbf{D}_{k, k}=\mathbf{D}^{O, k}+\mathbf{D}^{A_{1}, k}+\mathbf{D}^{A_{2}, k}+\mathbf{D}^{B_{1}, k}+\mathbf{D}^{B_{2}, k}+\mathbf{D}^{B_{3}, k} ; k=1, \ldots, K$

$\mathbf{D}_{k, k-1}=\mathbf{D}^{C_{1}, k}+\mathbf{D}^{C_{2}, k} ; k=2, \ldots, K$

$\mathbf{D}_{1, K}=\mathbf{D}^{F C_{1}, 1}+\mathbf{D}^{F C_{2}, 1}$

The stationary distribution has been worked out from the balance equations. These probabilities are equal to

$\boldsymbol{\pi}_{\mathbf{E}^{k}}=\pi_{\mathbf{E}^{1}} \mathbf{R}_{1, k}$, for $k=2, \ldots, K$,

being

$\mathbf{R}_{1, K}=\mathbf{D}_{1, K}\left(\mathbf{I}-\mathbf{D}_{K, K}\right)^{-1}$

$\mathbf{R}_{1, k}=\mathbf{R}_{1, k+1} \mathbf{D}_{k+1, k}\left(\mathbf{I}-\mathbf{D}_{k, k}\right)^{-1}$

for $k=2, \ldots, K-1$.

The vector $\pi_{\mathbf{E}^{1}}$ can be expressed as

$\boldsymbol{\pi}_{\mathbf{E}^{1}}=(1, \mathbf{0})\left(\left(\mathbf{I}+\sum_{k=2}^{K} \mathbf{R}_{1, k}\right) \mathbf{e} \mid\left[\mathbf{D}_{1,1}+\mathbf{R}_{1,2} \mathbf{D}_{2,1}-\mathbf{I}\right]^{*}\right)^{-1}$,

where the matrix $\mathbf{A}^{*}$ is the matrix $\mathbf{A}$ without the first column.

The stationary distribution associated to the macro-state $\mathbf{E}_{s}^{k}$ is given by the corresponding part of $\boldsymbol{\pi}_{\mathbf{E}^{k}}$ and it id denoted by $\boldsymbol{\pi}_{\mathbf{E}_{s}^{k}}$.

\subsection{Measures}

Several interesting reliability measures such as availability, reliability, mean times and mean number of events are calculated in this section for the transient and stationary regime. 


\subsubsection{Availability}

The availability is the probability that
system is operational at time $v$. It is given by

$A(v)=1-\sum_{k=1}^{K} \mathbf{p}_{E_{k}^{k}}^{v} \cdot \mathbf{e}$.

This measure is also calculated in the stationary case and it is equal to $A=1-\sum_{k=1}^{K} \pi_{E_{k}^{k}} \cdot \mathbf{e}$.

\subsubsection{Reliability}

Two different reliability functions have been built: the time up to the first time that the system in non-operational (all units in the repair facility) and the time up to the first time that the system is replaced (all units have undergone a non-repairable failure).

In the first case, the probability distribution is given by the phasetype distribution with representation $\left(\theta^{\prime}, \mathbf{D}^{\prime}\right)$ where the vector and the matrix are equal to $(\theta, \mathbf{D})$ restricted to the macro-states $E_{s}^{k}$ for $k=1, \ldots$, $K$ and $s=0, \ldots, k-1$.

In the second case, the time up to the first time that the system is replaced by another an identical one is phase-type distributed with representation $\left(\boldsymbol{\theta}, \mathbf{D}^{*}\right)$ where the matrix is given by $\mathbf{D}$ with the blocks $\mathbf{D}^{F C 1,1}=\mathbf{D}^{F C 2,1}=\mathbf{0}$.

\subsubsection{Mean time in each macro-state}

The mean time that the system is in macro-state $\mathbf{E}_{s}^{k}(k$ units in the system and $s$ of them in the repair facility) up to time $v$ is given by

$\psi_{k, s}(v)=\sum_{m=0}^{v} \mathbf{p}_{E_{s}^{k}}^{m} \cdot \mathbf{e}$.

From this expression, the mean time in macro state $\mathbf{E}^{k}(k$ units in the system) is given by

$\psi_{k}(v)=\sum_{s=0}^{k} \psi_{k, s}(v)$

The corresponding stationary values are $\psi_{k, s}=\sum_{m=0}^{v} \boldsymbol{\pi}_{E_{s}^{k}} \cdot \mathbf{e}$ and $\psi_{k}=$ $\sum_{s=0}^{k} \psi_{k, s}$.

\subsubsection{Mean operational time up to time v}

From the measures described above in Section 5.3.3, the mean time that the system is operational up to time $v$ can be calculated. It is given by

$\mu_{o p}(v)=\sum_{k=1}^{K} \sum_{s=0}^{k-1} \psi_{k, s}(v)$

This mean time in stationary regime is the operational time ratio and it is $\mu_{o p}=\sum_{k=1}^{K} \sum_{s=0}^{k-1} \psi_{k, s}$.

\subsubsection{Mean time that the repairpersons are idle and busy}

The systems proposed in this work have different number of repairpersons depending on the number of units in the system. One interesting aspect is to analyze the mean cumulative time that the repairpersons are idle up to a certain time. This measure is given by

$\mu_{\text {idle }}(\nu)=\sum_{k=1}^{K} \sum_{s=0}^{k-1}\left(\boldsymbol{R}_{k}-\min \left\{\boldsymbol{R}_{k}, s\right\}\right) \cdot \psi_{k, s}(\nu)$.

In the stationary regime this measure is the mean number of idle repairpersons per unit of time,

$\mu_{\text {idle_s }}=\sum_{k=1}^{K} \sum_{s=0}^{k-1}\left(\boldsymbol{R}_{k}-\min \left\{\boldsymbol{R}_{k}, s\right\}\right) \cdot \psi_{k, s}$.
Following a similar reasoning to analyze the number of repairpersons that are busy

$\mu_{\text {busy }}(v)=\sum_{k=1}^{K} \sum_{s=1}^{k} \min \left\{R_{k}, s\right\} \cdot \psi_{k, s}(v)$,

and in the stationary regime $\mu_{\text {busy_s }}=\sum_{k=1}^{K} \sum_{s=1}^{k} \min \left\{R_{k}, s\right\} \cdot \psi_{k, s}$.

\subsubsection{Mean time working on corrective and preventive repair}

The repairpersons can be working on corrective repair or preventive maintenance. The mean time that the repairpersons are working on corrective repair and preventive maintenance up to time $v$ is given respectively by

$\mu_{\text {corr }}(v)=\sum_{m=0}^{v} \sum_{k=1}^{K} \sum_{s=1}^{k} \mathbf{p}_{E_{s}^{k}}^{v} \cdot \mathbf{q}_{s}^{k}$

and

$\mu_{p m}(v)=\sum_{m=0}^{v} \sum_{k=1}^{K} \sum_{s=1}^{k} \mathbf{p}_{E_{s}^{k}}^{v} \cdot \mathbf{q}_{s}^{k}$,

where $\mathbf{q}_{s}^{k}(1)$ and $\mathbf{q}_{s}^{k}(2)$ are column vectors that contains the number of repairpersons that are working on corrective repair and preventive maintenance respectively according to the macro-state $E_{s}^{k}$. These column vectors are given by

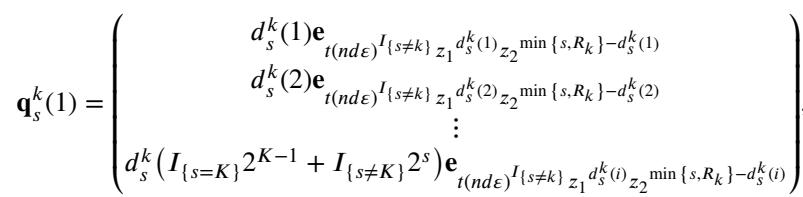

for

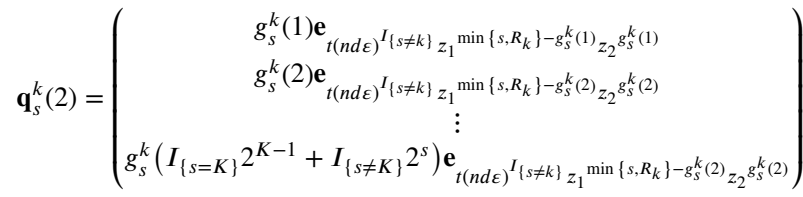

being $d_{s}^{k}(i)$ the $i$ th element of the vector $\mathbf{d}_{s}^{k}=$

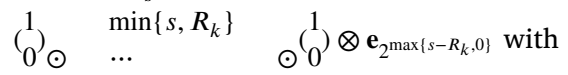

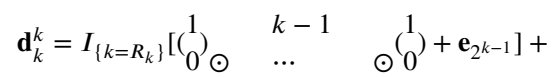

$I_{\left\{k>R_{k}\right\}}\left(\begin{array}{lll}1 & 1\end{array}\right) \quad \begin{array}{ll}R_{k} & \cdots\end{array} \quad \odot\left(\begin{array}{l}1 \\ 0\end{array}\right) \otimes \mathbf{e}_{2^{k-R_{k}-1}} \quad$ and $\quad g_{s}^{k}(i) \quad$ the $i$-th element

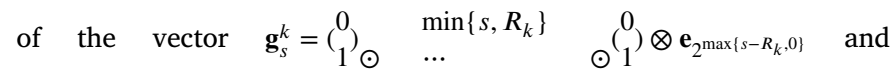

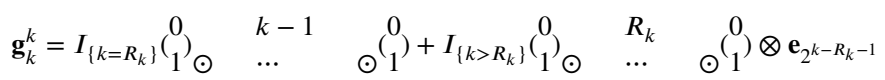
for $k=1, \ldots, K$ and $s=1, \ldots, k$ where $\mathbf{a} \odot \mathbf{b}=\mathbf{a} \otimes \mathbf{e}_{m}+\mathbf{e}_{n} \otimes \mathbf{a}$ being $\mathbf{a}$ and b column vectors with order $n$ and $m$ respectively.

These measures in stationary regime are $\mu_{\text {corr } s}=$ $\sum_{k=1}^{K} \sum_{s=1}^{k} \pi_{E_{s}^{k}} \cdot \mathbf{q}_{s}^{k}(1)$ and $\mu_{p m_{-} s}=\sum_{k=1}^{K} \sum_{s=1}^{k} \pi_{E_{s}^{k}} \cdot \mathbf{q}_{s}^{k}(2)$ respectively.

\subsubsection{Mean number of events}

Thanks to the structure built, the expected number of events up to a certain time $v$ is worked out. It is given by $\Lambda^{Y}(v)=\sum_{u=1}^{v} \mathbf{p}^{u-1} \mathbf{D}^{Y} \mathbf{e}$, for $Y=A_{1}, A_{2}, B_{1}, B_{2}, B_{3}, C_{1}, C_{2} F C_{1}, F C_{2}$. In stationary regime, the mean number of events per unit of time is $\Lambda^{Y}=\pi \mathbf{D}^{Y} \mathbf{e}$.

\section{Costs and rewards}

Several costs and rewards have been included in the model to study the effectiveness of the model from an economic standpoint. Thus, we assume that there is a gross profit per unit of time while the system is operational equal to $B$. While the system is operational a mean cost 
per unit of time depending on the operational phase occurs. This cost is given by the vector $\boldsymbol{c}_{\mathbf{0}}$. There are two different types of repair, corrective repair and preventive maintenance. The mean cost per unit of time when a unit is in corrective repair or preventive maintenance depending on the repair phase is given by the vectors $c \boldsymbol{r}_{1}$ and $\boldsymbol{c r} \boldsymbol{r}_{2}$ respectively. Also, we assume a fixed cost per unit of time for each repairperson equal to $H$ and a loss per unit of time while the system is not operational equal to $C$. Finally, each time that the online unit undergoes a repairable failure or a major inspection a fixed cost is produced equal to $f c r$ or fpm respectively. The mean cost per one new unit is fnu (the cost of a new system is $K \cdot f n u$ ).

To calculate the total net profit up to time $v$ is necessary to build the vector cost for the macro-state $E_{s}^{k}$ and several rewards and costs functions.

\subsection{Net profit vector associated to the phases}

When the systems is composed of $k$ units and $s$ of them are in the repair facility, then the online unit provokes a net reward for the different phases of the system given by

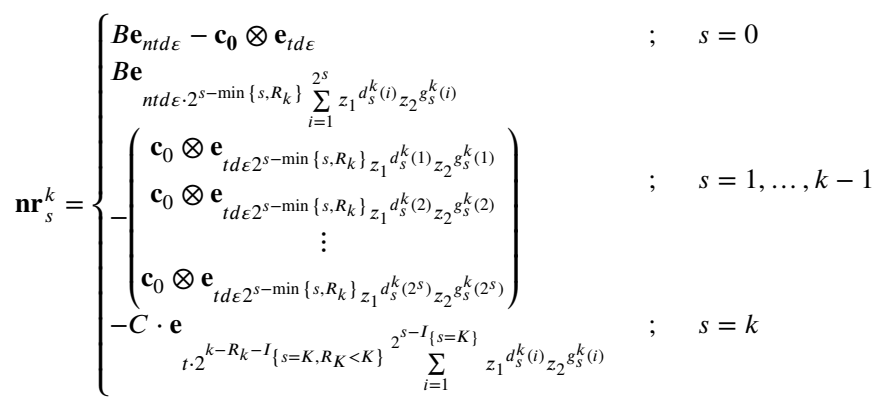

Then, for the state space it is

$\mathbf{n r}=\left(\mathbf{n r}_{0}^{K^{\prime}}, \mathbf{n r}_{1}^{K^{\prime}}, \ldots, \mathbf{n r}_{K}^{K^{\prime}}, \mathbf{n r}_{0}^{K^{\prime}}, \mathbf{n r}_{1}^{K-1^{\prime}}, \ldots, \mathbf{n r}_{K-1}^{K-1^{\prime}}, \ldots, \mathbf{n r}_{0}^{1^{\prime}}, \mathbf{n r}_{1}^{1^{\prime}}\right)^{\prime}$.

If the repair facility is considered, the cost vector per unit of time depending on the type of repair associated to the macro-state $E_{s}^{k}$ for $s=1, \ldots, k$, is

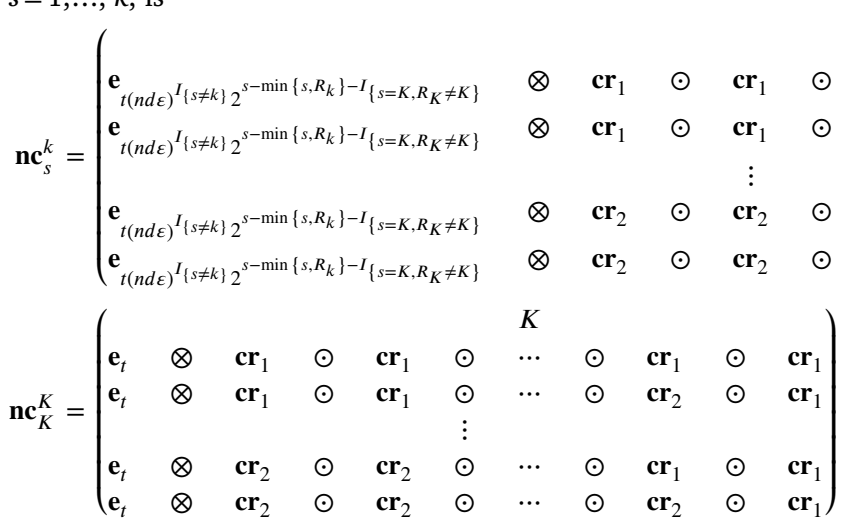

The total vector for the cost due to repair is given by

$$
\mathbf{n c}=\left(\mathbf{n c}_{0}^{K^{\prime}}, \mathbf{n c}_{1}^{K^{\prime}}, \ldots, \mathbf{n c}_{K}^{K^{\prime}}, \mathbf{n c}_{0}^{K^{\prime}}, \mathbf{n c}_{1}^{K-1^{\prime}}, \ldots, \mathbf{n c}_{K-1}^{K-1^{\prime}}, \ldots, \mathbf{n c}_{0}^{1^{\prime}}, \mathbf{n c}_{1}^{\mathbf{1}^{\prime}}\right)^{\prime} .
$$

Thus, the net profit vector associated to the macro-state $E_{s}^{k}$ is given by $\mathbf{c}_{0}^{k}=\mathbf{n r}_{0}^{k}, \mathbf{c}_{s}^{k}=\mathbf{n r}_{s}^{k}-\mathbf{n c}_{s}^{k}$ for $s=1, \ldots, k$. Finally, the net column profit vector associated to the macro-state $E^{k}$ is given by $\mathbf{c}^{k}=\left(\mathbf{c}_{0}^{k}, \ldots, \mathbf{c}_{k}^{k}\right)^{\prime}$ thus the global net column profit vector associated to the macro-state $E$ is given by

$\mathbf{c}=\mathbf{n r}-\mathbf{n c}=\left(\begin{array}{c}\mathbf{c}^{K} \\ \mathbf{c}^{K-1} \\ \vdots \\ \mathbf{c}^{1}\end{array}\right)$

\subsection{Rewards measures}

Several rewards measures have been built in transient and stationary regime.

Mean net profit up to time $v$

The mean net profit by considering only the online unit up to time $v$ is given by

$\Phi_{w}^{v}=\sum_{m=0}^{v} \mathbf{p}^{m} \cdot \mathbf{n r}$,

and it is in stationary regime the meat neat profit per unit of time, $\Phi_{w s}=$ $\boldsymbol{\pi} \cdot \mathbf{n r}$.

Mean cost due to corrective and preventive repair

The mean cost due to corrective repair and preventive maintenance up to time up $v$ is given respectively by

$\Phi_{c r}^{v}=\sum_{m=0}^{v} \mathbf{p}^{m} \cdot \mathbf{m} \mathbf{c}^{c r}$ and $\Phi_{p m}^{v}=\sum_{m=0}^{v} \mathbf{p}^{m} \cdot \mathbf{m c}^{p m}$ where $\mathbf{m} \mathbf{c}^{c r}$ is the vector nc with $\mathbf{c r}_{2}=\mathbf{0}_{\mathrm{z}_{2}}$ and $\mathbf{m} \mathbf{c}^{p m}$ is the vector $\mathbf{n c}$ with $\mathbf{c r}_{1}=\mathbf{0}_{\mathrm{z}_{1}}$, being $\mathbf{0}_{a}$ a column vector of zeros with order $a$.

These measures in stationary regime, net cost per unit of time due to corrective or preventive maintenance, are $\Phi_{c r_{-} s}=\boldsymbol{\pi} \cdot \mathbf{m c}^{c r}$ and $\Phi_{p m_{-} s}=$ $\boldsymbol{\pi} \cdot \mathbf{m c}^{p m}$ respectively.

Total net profit

The total net profit up to time $v$ is worked out by adding costs and profits produced by the events. If the fixed cost per event is included then it is equal to

$\Phi^{v}=\Phi_{w}^{v}-\Phi_{c r}^{v}-\Phi_{p m}^{v}-\left(1+\Lambda^{F C 1}(v)+\Lambda^{F C 2}(v)\right) \cdot K \cdot f n u-\left(\Lambda^{A 1}(v)\right.$

$\left.+\Lambda^{A 2}(v)\right) \cdot f c r-\left(\Lambda^{B 1}(v)+\Lambda^{B 2}(\nu)+\Lambda^{B 3}(v)\right) \cdot f p m-\left(\mu_{\text {idle }}+\mu_{\text {busy }}\right) \cdot H$.

Finally, the total net profit per unit of time (stationary regime) is

$\Phi_{s}=\Phi_{w_{-} s}-\Phi_{c r_{-} s}-\Phi_{p m_{-} s}-\left(1+\Lambda^{F C 1}+\Lambda^{F C 2}\right) \cdot K \cdot f n u$

$-\left(\Lambda^{A 1}+\Lambda^{A 2}\right) \cdot f c r-\left(\Lambda^{B 1}+\Lambda^{B 2}+\Lambda^{B 3}\right) \cdot f p m-\left(\mu_{\text {idle }}+\mu_{\text {busy }}\right) \cdot H$.

\section{A numerical example}

Any facility that requires a reliable electrical supply (such as department stores, hospitals, military installations and hydroelectric plants) must have additional generating resources available. When the ordinary electricity supply fails, a cold standby generating set comes into action. For a large dam, at least two such generating sets must be installed in cold standby. The generating set may fail for the same reasons as any motor, provoking either a total failure of the motor or a repairable failure, and preventive maintenance may be necessary. Therefore, we assume a cold standby system composed of three units, as Systems II and III. To optimize the system, two questions must be answered. Is preventive maintenance profitable? How many repairpersons, depending on the number of units in the system, would have to be deployed to optimize the profit? In this numerical example, the effectiveness of preventive maintenance is analyzed and the optimum number of repairpersons is calculated. 

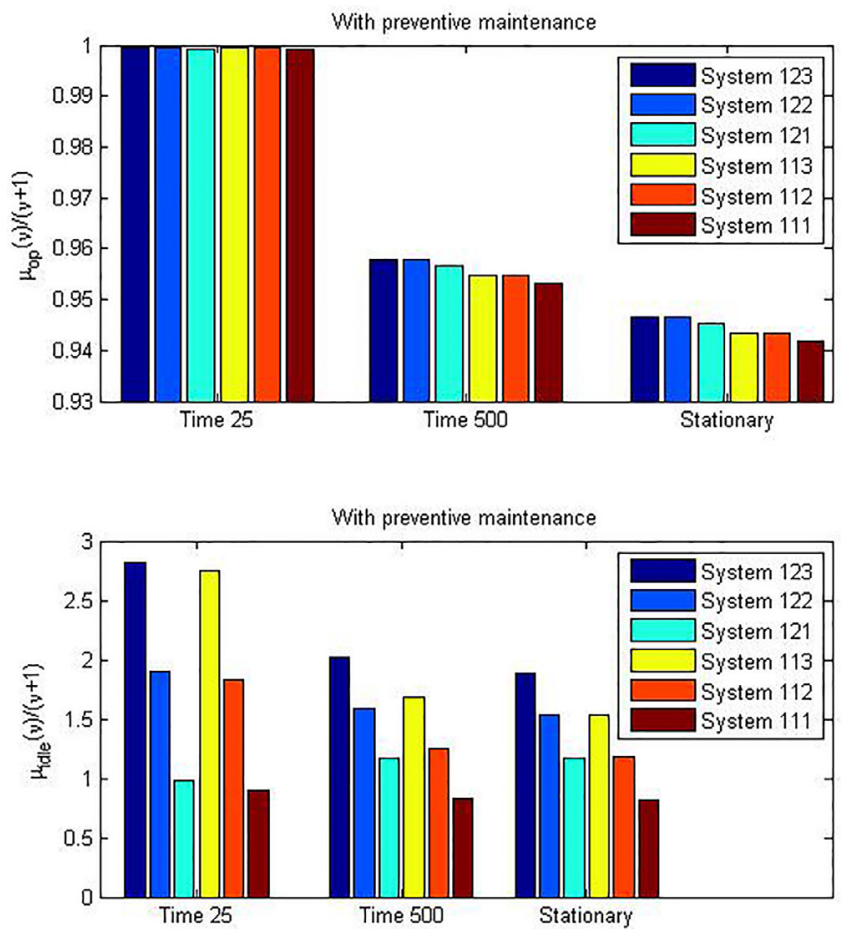
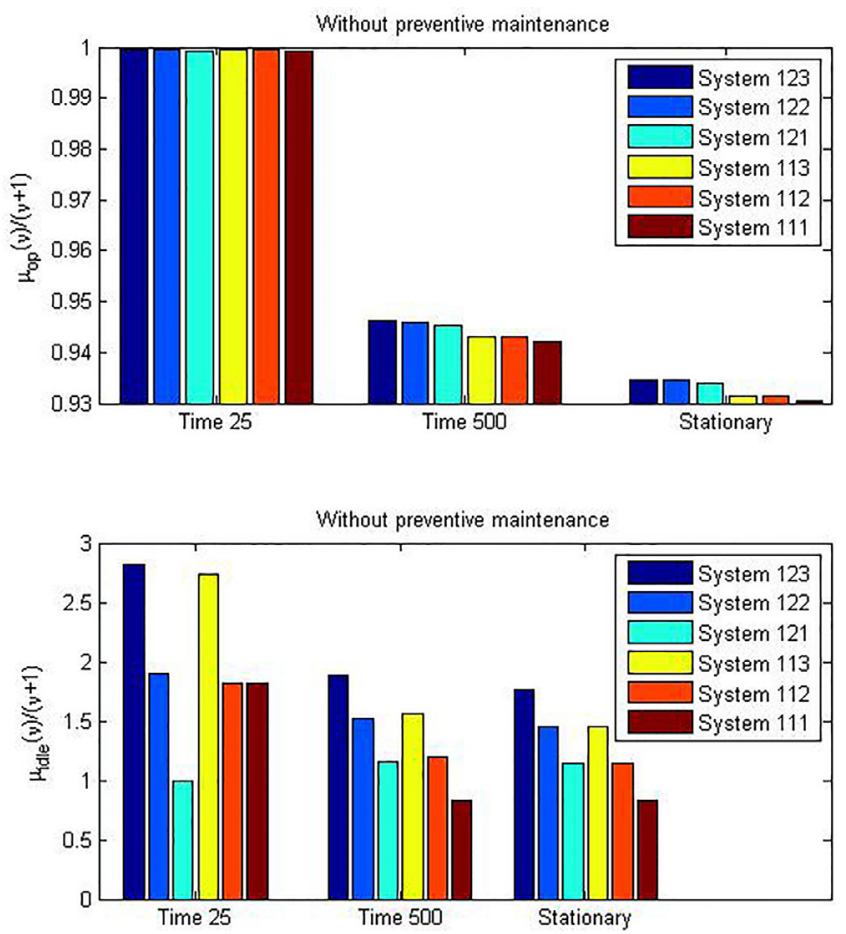

Fig. 3. Mean operational ratio (first row) and mean number of idle repairpersons per unit of time (second row) with preventive maintenance (first column) and without preventive maintenance (second column).

Table 1

Internal operational, external shock and inspection time distributions.

\begin{tabular}{|c|c|c|c|c|c|c|c|c|c|c|}
\hline \multicolumn{6}{|c|}{ Internal operational time } & \multicolumn{2}{|c|}{ External shock } & \multicolumn{3}{|c|}{ Inspection time } \\
\hline \multicolumn{6}{|c|}{$\boldsymbol{\alpha}=(1,0,0,0,0)$} & \multicolumn{2}{|l|}{$\boldsymbol{\gamma}=(1,0)$} & \multicolumn{3}{|c|}{$\boldsymbol{\eta}=(1,0)$} \\
\hline & 0.99 & 0.002 & 0 & 0 & 0 & & & & & \\
\hline & 0 & 0.9 & 0.001 & 0 & 0 & & & & & \\
\hline \multirow[t]{3}{*}{$\mathbf{T}=$} & 0 & 0 & 0.9 & 0.002 & 0 & $\mathbf{L}=\left(\begin{array}{c}0.89 \\
01\end{array}\right.$ & $\left.\begin{array}{c}0.1 \\
0.8\end{array}\right)$ & $\mathbf{M}=$ & $\begin{array}{l}0.85 \\
0.45\end{array}$ & $\left.\begin{array}{l}0.1 \\
0.4\end{array}\right)$ \\
\hline & 0 & 0 & 0 & 0.6 & 0 & & & & & \\
\hline & 0 & 0 & 0 & 0 & $0.6)$ & & & & & \\
\hline \multicolumn{6}{|c|}{ Mean time: 102.0201} & \multicolumn{2}{|c|}{ Mean time: 25} & \multicolumn{3}{|c|}{ Mean time: 15.56} \\
\hline
\end{tabular}

\section{System times}

The internal behavior of the online unit passes through five performance levels, where the degradation is minor in the first three stages and major in the last two. The online unit is also subject to external shocks and inspections. The operational time distribution of the online unit, the inspection time distribution and the external shock time are PH distributed with representation given in Table 1.

Each time that the online unit undergoes an external shock, a total non-repairable failure occurs with a probability equal to 0.05 . If no such failure occurs, the internal performance may be degraded according to the following probability matrix

$\mathbf{W}=\left(\begin{array}{ccccc}0.6 & 0.2 & 0.1 & 0.1 & 0 \\ 0 & 0.6 & 0.2 & 0.1 & 0.1 \\ 0 & 0 & 0.6 & 0.2 & 0.2 \\ 0 & 0 & 0 & 0.5 & 0.3 \\ 0 & 0 & 0 & 0 & 0.4\end{array}\right)$

When an external shock takes place, cumulative external damage occurs. Four external degradation levels are assumed, the first two of which are minor and the last two, major. Changes in the external degra- dation levels are governed by the matrix

$\mathbf{D}=\left(\begin{array}{cccc}0 & 0.3 & 0.7 & 0 \\ 0 & 0 & 0.6 & 0.4 \\ 0 & 0 & 0 & 0.5 \\ 0 & 0 & 0 & 0.3\end{array}\right)$,

where initially the external degradation level is the stage 1 (without external damage).

Each time that a repairable failure or a major inspection occurs, the online units goes to the repair facility. The corrective repair time and the preventive maintenance time distributions are given in Table 2. Performance of the systems according to the number of repairpersons

As mentioned above, the systems with and without preventive maintenance (Systems III and II, respectively) are compared by considering all possibilities for the number of repairpersons. Thus, the system $i j_{-} k$ denotes a system with $i, j, k$ repairpersons when there are $1,2,3$ units in the system respectively for $i=1, j=1,2, k=1,2,3$. In total there are 12 possible systems, six with preventive maintenance and six without. Several measures have been worked out and compared in transient and stationary regime.

Fig. 3 shows the mean operational time and the mean number of idle repairpersons per unit of time for Systems II and III. The optimum mean operational time ratio is reached for System 1_2_3 when the operational time ratio in a stationary regime is equal to 0.9467 for the 

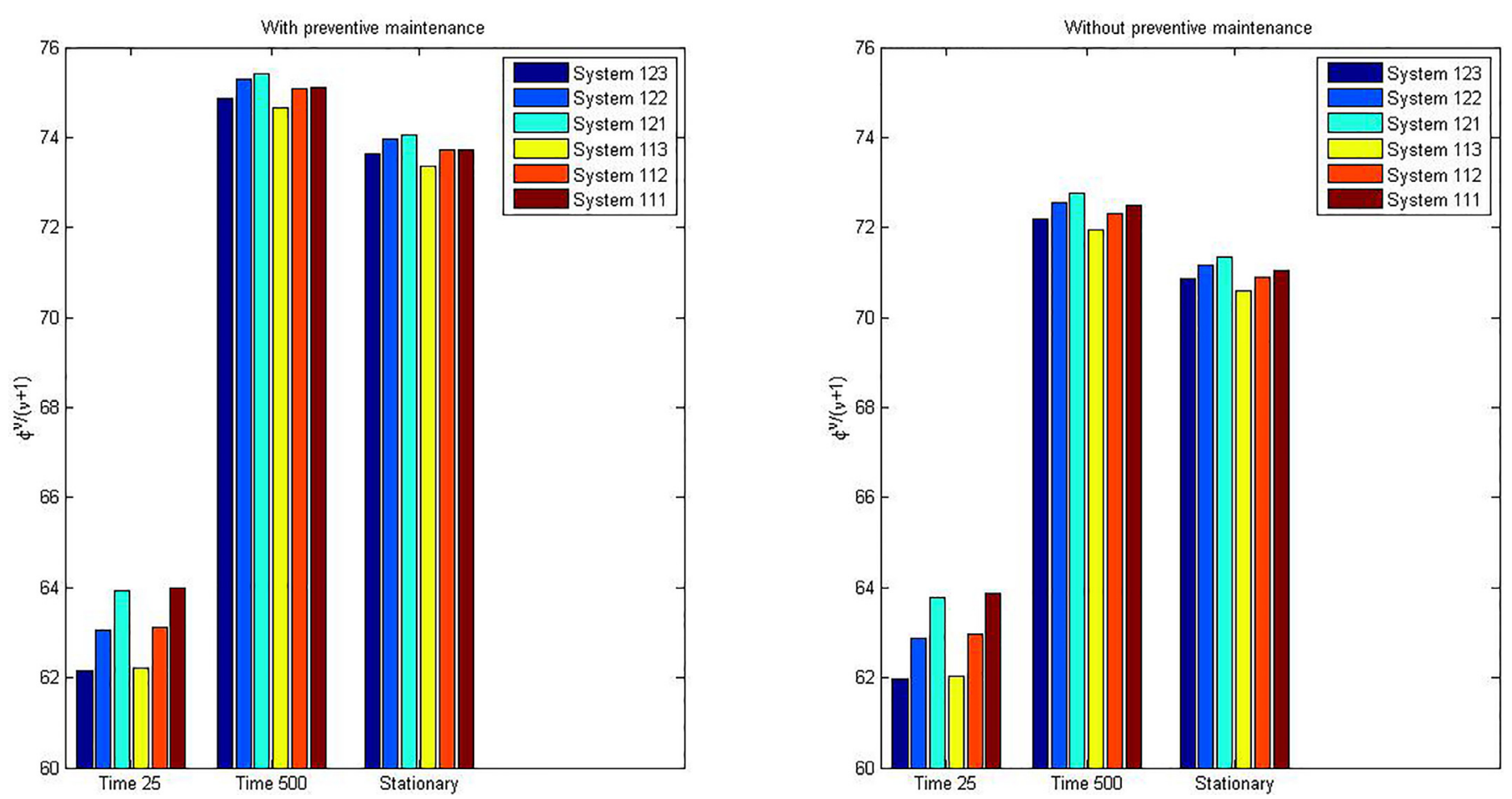

Fig. 4. Mean net profit per unit of time up to time 25,500 and the stationary case for system II (with preventive maintenance) and system III (without preventive maintenance).

Table 2

Corrective repair and preventive maintenance time distributions.

\begin{tabular}{ll}
\hline Corrective repair time distribution & Preventive maintenance time distribution \\
\hline $\boldsymbol{\beta}^{1}=(1,0)$ & $\boldsymbol{\beta}^{2}=(1,0)$ \\
$\mathbf{S}_{1}=\left(\begin{array}{cc}0.91 & 0.01 \\
0 & 0.8\end{array}\right)$ & $\mathbf{S}_{2}=\left(\begin{array}{cc}0.1 & 0.1 \\
0 & 0.1\end{array}\right)$ \\
Mean time: 11.67 & Mean time: 1.23 \\
\hline
\end{tabular}

Table 3

Mean number of events up to time 1500 (without preventive maintenance in parenthesis).

\begin{tabular}{|c|c|c|c|c|c|c|c|c|c|}
\hline SYSTEM & & $\Lambda^{A_{1}}(1500)$ & $\Lambda^{A_{2}}(1500)$ & $\Lambda^{B_{1}}(1500)$ & $\Lambda^{B_{2}}(1500)$ & $\Lambda^{B_{3}}(1500)$ & $\Lambda^{C_{1}}(1500)$ & $\Lambda^{C_{2}}(1500)$ & $\begin{array}{l}\Lambda^{F C_{1}}(1500) \\
+\Lambda^{F C_{2}}(1500)\end{array}$ \\
\hline \multirow[t]{6}{*}{ Time 1500} & $1 \_2 \_3$ & $21.6051(22.3598)$ & $0.0160(0.0158)$ & $0.0741(-)$ & $12.1965(-)$ & $0.2521(-)$ & $3.5750(3.1678)$ & 5.6187 (8.5369) & $4.1283(5.3222)$ \\
\hline & $1 \_2 \_2$ & $21.6038(22.3585)$ & $0.0160(0.0158)$ & $0.0741(-)$ & $12.1957(-)$ & $0.2521(-)$ & $3.5748(3.1676)$ & $5.6184(8.5365)$ & $4.1280(5.3219)$ \\
\hline & $1 \_2 \_1$ & $21.5840(22.3403)$ & $0.0160(0.0157)$ & $0.0733(-)$ & $12.1253(-)$ & $0.2499(-)$ & $3.5703(3.1651)$ & $5.6237(8.5298)$ & $4.1283(5.3177)$ \\
\hline & 1_1_3 & $21.5328(22.2864)$ & $0.0159(0.0157)$ & $0.0738(-)$ & $12.1531(-)$ & $0.2512(-)$ & 3.5634 (3.1577) & $5.6012(8.5098)$ & $4.1136(5.3037)$ \\
\hline & 1_1_2 & $21.5315(22.2850)$ & $0.0159(0.0157)$ & $0.0738(-)$ & $12.1522(-)$ & $0.2512(-)$ & $3.5632(3.1575)$ & $5.6009(8.5093)$ & $4.1133(5.3034)$ \\
\hline & 1_1_1 & $21.5053(22.2638)$ & $0.0159(0.0157)$ & $0.0730(-)$ & $12.0797(-)$ & $0.2489(-)$ & $3.5577(3.1546)$ & $5.6045(8.5016)$ & $4.1123(5.2985)$ \\
\hline
\end{tabular}

system with preventive maintenance and 0.9346 for the case without preventive maintenance. This outcome is to expect but as the repairpersons have a cost, it is interesting to analyze the mean number of idle repairpersons. In this case, the maximum is reached for System 1_2_3 with a mean number of idle repairpersons per unit of time in the stationary regime equal to 1.8847 and 1.7663 for the systems with and without preventive maintenance, respectively.

Another interesting aspect to study is that of the mean number of events up to a certain time. This measure was calculated for every system and for several units of time. Table 3 shows the results obtained for 1500 units of time.
The number of new systems up to time 1500 is given by the last column of the Table 3. The minimum is reached when always one repairperson is assumed.

Analysis of systems when costs and rewards are included

Rewards and costs have been included in the analysis to optimize the model form an economical standpoint. Each time that the system is operational a reward equal to $B=100$ is produced and a lost with the same quantity is considered while the system is not operational. The operational cost per unit of time while the online unit is working depends on the internal degradation level according to the vector $\mathbf{c}_{0}=$ $(10,20,30,40,50)$. While the unit is being repaired two different costs per unit of time can be produced according if they are corrective repair or preventive maintenance. In the first case a cost equal to 5 is given and 
in the second case 0.5 . Finally when a repairable failure occurs a fixed cost equal to 20 is produced and if it is a major inspection this cost is 1 . A new unit of the system costs 200 and any repairperson has a cost of one per unit of time.

The mean net reward has been calculated for any system to achieve the more profitable system. Fig. 4 shows them per unit of time for the cases with and without preventive maintenance.

If the mean net profit is observed in stationary regime the most profitable situation is for system 1_2_1 with preventive maintenance. Initially the number of repairpersons should be only one, when the first non-repairable failure occurs then one repairperson is added and finally only one repairperson should be when another non-repairable failure occurs. The optimum mean net profit in this case is equal to 74.0513 in stationary regime.

\section{Conclusions}

In this study, three multi-state cold standby systems, evolving in discrete time, are modeled in an algorithmic and computational form using Markovian arrival processes with marked arrivals. The online unit is a multi-state device depending on degradation/performance levels. The three systems are modeled following similar methods, ranked from simplest to most complex. The latter includes multiple events: internal failure, external shocks with different consequences and inspections. Corrective repair and preventive maintenance are included as responses to a repairable failure and to major damage (internal or external) when the unit is inspected, respectively. Non-repairable failures, whether internal or due to an external shock, are possible and in this case the unit is removed.

Two interesting contributions are made in the present study. The number of repairpersons is indeterminate and variable depending on the number of units in the system. A system can be optimized by considering two different standpoints: the profitability of preventive maintenance and the number of repairpersons present according to the number of units in the system.

This complex system is modeled by a MMAP, which is shown to be useful for expressing the modeling and its associated measures in a wellstructured form. Furthermore, this method makes it possible to determine the transient and stationary distributions and measures associated with the system in a matrix-algorithmic and computational form.

Other redundant systems such as warm standby systems and $k$-outof- $n$ : $G$ systems can be modeled following this algorithmic methodology. Also, in a similar way and following this methodology, repairpersons could be replaced by repair sources, a situation in which costs and the associated repair times need not be the same.

Several measures, developed in an algorithmic form, are worked out in transient and stationary regime in an algorithmic and computational way. A numerical example illustrates the versatility of the modeling performed, and the optimum system is obtained.

\section{Acknowledgments}

This paper is partially supported by the Junta de Andalucía, Spain, under the grant FQM-307 and by the Ministerio de Economía y Competitividad, España, under Grant MTM2013-47929-P and by the European Regional Development Fund (ERDF).

\section{Appendix A}

\section{MODEL I}

The transition matrices for the online unit for the system I case are,

$O:$ No events: $\mathbf{H}_{0}=\mathbf{T}$

$A_{1}$ : Repairable internal failure: $\mathbf{H}_{\text {rep }}=\mathbf{T}_{r}^{0} \boldsymbol{\alpha} ; \mathbf{H}_{\text {rep }}^{\prime}=\mathbf{T}_{r}^{0}$

$C_{1}$ : Non-repairable failure: $\mathbf{H}_{\mathrm{nrep}}=\mathbf{T}_{n r}^{0} \boldsymbol{\alpha} ; \mathbf{H}_{\mathrm{nrep}}^{\prime}=\mathbf{T}_{n r}^{0}$
MODEL II

The transition matrices for the online unit for the system II case are,

$O:$ No events: $\mathbf{H}_{0}=\mathbf{T} \otimes \mathbf{L} \otimes \mathbf{I}+\mathbf{T W} \otimes \mathbf{L}^{0} \boldsymbol{\gamma} \otimes \mathbf{D}\left(1-\omega^{0}\right)$

$A$ : Repairable internal failure

$A_{1}$ : Repairable internal failure not due to shock:

$$
\begin{aligned}
& \mathbf{H}_{\mathrm{rep}}^{1}=\mathbf{T}_{r}^{0} \boldsymbol{\alpha} \otimes \mathbf{L} \otimes \mathbf{e} \omega+\mathbf{T}_{r}^{0} \boldsymbol{\alpha} \otimes \mathbf{L}^{0} \boldsymbol{\gamma} \otimes \mathbf{D e} \omega\left(1-\omega^{0}\right) \\
& \mathbf{H}_{\mathrm{rep}}^{\prime 1}=\mathbf{T}_{r}^{0} \otimes \mathbf{L} \otimes \mathbf{e}_{d}+\mathbf{T}_{r}^{0} \otimes \mathbf{L}^{0} \boldsymbol{\gamma} \otimes \mathbf{D e}\left(1-\omega^{0}\right)
\end{aligned}
$$

$A_{2}$ : Repairable internal failure due to shock: $\mathbf{H}_{\text {rep }}^{2}=\mathbf{T W}^{0} \boldsymbol{\alpha} \otimes \mathbf{L}^{0} \boldsymbol{\gamma} \otimes$ $\operatorname{De} \omega\left(1-\omega^{0}\right)$

$\mathbf{H}_{\text {rep }}^{2}=\mathbf{T W}^{0} \otimes \mathbf{L}^{0} \boldsymbol{\gamma} \otimes \mathbf{D e}\left(1-\omega^{0}\right)$

$C$ : Non-repairable failure

$C_{1}$ : Non-repairable internal failure: $\mathbf{H}_{\mathrm{nrep}}^{1}=\mathbf{T}_{n r}^{0} \boldsymbol{\alpha} \otimes \mathbf{L} \otimes \mathbf{e} \boldsymbol{\omega}+\mathbf{T}_{n r}^{0} \boldsymbol{\alpha} \otimes$

$$
\mathbf{L}^{0} \boldsymbol{\gamma} \otimes \operatorname{De} \omega\left(1-\omega^{0}\right)
$$

$$
\mathbf{H}_{\text {nrep }}^{\prime 1}=\mathbf{T}_{n r}^{0} \otimes \mathbf{L} \otimes \mathbf{e}+\mathbf{T}_{n r}^{0} \otimes \mathbf{L}^{0} \boldsymbol{\gamma} \otimes \mathbf{D e}\left(1-\omega^{0}\right)
$$

$C_{2}$ : Non-repairable failure due to shock: $\mathbf{H}_{\text {nrep }}^{2}=\mathbf{e \alpha} \otimes \mathbf{L}^{0} \boldsymbol{\gamma} \otimes$ $\left(\mathbf{e} \omega \omega^{0}+\mathbf{D}^{0} \boldsymbol{\omega}\left(1-\omega^{0}\right)\right)$

$\mathbf{H}_{\text {nrep }}^{2}=\mathbf{e} \otimes \mathbf{L}^{0} \boldsymbol{\gamma} \otimes\left(\mathbf{e} \omega^{0}+\mathbf{D}^{0}\left(1-\omega^{0}\right)\right)$

MODEL III

Auxiliary matrices for minor/major inspection

The matrix $\mathbf{U}_{l}$ and $\mathbf{V}_{l}$, for $l=1,2$, are square matrices of order $n$ and $d$ respectively, whose element $(s, t)$ is given by,

$U_{1}(s, t)=\left\{\begin{array}{lll}1 & ; & 1 \leq s=t \leq n_{1} \\ 0 & ; & \text { otherwise }\end{array}, U_{2}(s, t)=\left\{\begin{array}{lll}1 & ; & s=t>n_{1} \\ 0 & ; & \text { otherwise }\end{array}\right.\right.$
$V_{1}(s, t)=\left\{\begin{array}{lll}1 & ; & 1 \leq s=t \leq d_{1} \\ 0 & ; & \text { otherwise }\end{array}, V_{2}(s, t)=\left\{\begin{array}{lll}1 & ; & s=t>d_{1} \\ 0 & ; & \text { otherwise }\end{array}\right.\right.$

The matrices $\mathbf{U}$ and $\mathbf{V}$ will be taken into account when one inspection occurs and the internal degradation level and cumulative external damage are observed respectively. The subscripts 1 and 2 will be considered when the damage observed is minor or major respectively.

$O$ : No events:

$$
\begin{aligned}
\mathbf{H}_{0}= & {\left[\mathbf{T} \otimes \mathbf{L} \otimes \mathbf{I}+\mathbf{T} \mathbf{W} \otimes \mathbf{L}^{0} \boldsymbol{\gamma} \otimes \mathbf{D}\left(1-\omega^{0}\right)\right] \otimes \mathbf{M} } \\
& +\left[\mathbf{U}_{1} \mathbf{T} \otimes \mathbf{L} \otimes \mathbf{V}_{1}+\mathbf{U}_{1} \mathbf{T W} \otimes \mathbf{L}^{0} \boldsymbol{\gamma} \otimes \mathbf{V}_{1} \mathbf{D}\left(1-\omega^{0}\right)\right] \otimes \mathbf{M}^{0} \eta \\
\mathbf{H}_{\mathrm{mr}}^{\prime}= & {\left[\mathbf{U}_{1} \mathbf{T} \otimes \mathbf{L} \otimes \mathbf{V}_{2} \mathbf{I}+\mathbf{U}_{2} \mathbf{T} \otimes \mathbf{L} \otimes \mathbf{I}\right.} \\
& +\mathbf{U}_{1} \mathbf{T W} \otimes \mathbf{L}^{0} \boldsymbol{\gamma} \otimes \mathbf{V}_{2} \mathbf{D}\left(1-\omega^{0}\right) \\
& \left.+\mathbf{U}_{2} \mathbf{T W} \otimes \mathbf{L}^{0} \boldsymbol{\gamma} \otimes \mathbf{D}\left(1-\omega^{0}\right)\right] \otimes \mathbf{M}^{0} \eta
\end{aligned}
$$

$B$ : Major revision

$B_{1}$ : Major revision for only internal major damage

$$
\begin{aligned}
\mathbf{H}_{\mathrm{mr}}^{1}= & {\left[\mathbf{U}_{2}\left(\mathbf{e}-\mathbf{T}^{0}\right) \boldsymbol{\alpha} \otimes \mathbf{L} \otimes \mathbf{V}_{1} \mathbf{e \omega}\right.} \\
& \left.+\mathbf{U}_{2} \mathbf{T W e} \boldsymbol{\alpha} \otimes \mathbf{L}^{0} \boldsymbol{\gamma} \otimes \mathbf{V}_{1} \operatorname{De} \boldsymbol{\omega}\left(1-\omega^{0}\right)\right] \otimes \mathbf{M}^{0} \eta
\end{aligned}
$$

$B_{2}$ : Major revision for only external cumulative damage

$\mathbf{H}_{\mathrm{mr}}^{2}=\left[\mathbf{U}_{1}\left(\mathbf{e}-\mathbf{T}^{0}\right) \boldsymbol{\alpha} \otimes \mathbf{L} \otimes \mathbf{V}_{2} \mathbf{e} \boldsymbol{\omega}+\mathbf{U}_{1} \mathbf{T W e} \boldsymbol{\alpha} \otimes \mathbf{L}^{0} \boldsymbol{\gamma} \otimes \mathbf{V}_{2} \mathbf{D e} \boldsymbol{\omega}\left(1-\omega^{0}\right)\right] \otimes \mathbf{M}^{0} \eta$

$B_{3}$ : Major revision for internal and external cumulative damage

$\mathbf{H}_{\mathrm{mr}}^{3}=\left[\mathbf{U}_{2}\left(\mathbf{e}-\mathbf{T}^{0}\right) \boldsymbol{\alpha} \otimes \mathbf{L} \otimes \mathbf{V}_{2} \mathbf{e} \boldsymbol{\omega}+\mathbf{U}_{2} \mathbf{T W e} \boldsymbol{\alpha} \otimes \mathbf{L}^{0} \boldsymbol{\gamma} \otimes \mathbf{V}_{2} \mathbf{D e \omega}\left(1-\omega^{0}\right)\right] \otimes \mathbf{M}^{0} \eta$

$C$ : Non-repairable failure

$C_{1}$ : Non-repairable internal failure:

$$
\begin{aligned}
& \mathbf{H}_{\text {nrep }}^{1}=\mathbf{T}_{n r}^{0} \boldsymbol{\alpha} \otimes \mathbf{L} \otimes \mathbf{e \omega} \otimes \mathbf{e} \boldsymbol{\eta}+\mathbf{T}_{n r}^{0} \boldsymbol{\alpha} \otimes \mathbf{L}^{0} \boldsymbol{\gamma} \otimes \mathbf{D e \omega}\left(1-\omega^{0}\right) \otimes \mathbf{e} \eta \\
& \mathbf{H}_{\text {nrep }}^{\prime 1}=\mathbf{T}_{n r}^{0} \otimes \mathbf{L} \otimes \mathbf{e} \otimes \mathbf{e}+\mathbf{T}_{n r}^{0} \otimes \mathbf{L}^{0} \boldsymbol{\gamma} \otimes \mathbf{D e}\left(1-\omega^{0}\right) \otimes \mathbf{e}
\end{aligned}
$$

$C_{2}$ : Non-repairable failure due to shock: $\mathbf{H}_{\text {nrep }}^{2}=\mathbf{e} \boldsymbol{\alpha} \otimes \mathbf{L}^{0} \boldsymbol{\gamma} \otimes$ $\left(\mathbf{e} \omega \omega^{0}+\mathbf{D}^{0} \boldsymbol{\omega}\left(1-\omega^{0}\right)\right) \otimes \mathbf{e} \eta$

$$
\mathbf{H}_{\text {nrep }}^{\prime 2}=\mathbf{e} \otimes \mathbf{L}^{0} \boldsymbol{\gamma} \otimes\left(\mathbf{e} \omega^{0}+\mathbf{D}^{0}\left(1-\omega^{0}\right)\right) \otimes \mathbf{e}
$$




\section{Appendix B}

The matrices for the Markovian arrival processes have been developed in the following way.

$\mathbf{D}^{Y}=\operatorname{diag}\left(\mathbf{D}^{Y, K}, \mathbf{D}^{Y, K-1}, \mathbf{D}^{Y, K-2}, \ldots, \mathbf{D}^{Y, 1}\right)$

for $Y=O, A_{1}, A_{2}, B_{1}, B_{2}$ and

$\mathbf{D}^{Y}=\left(\begin{array}{lllll}\mathbf{0} & \mathbf{D}^{Y, K} & & & \\ & \mathbf{0} & \mathbf{D}^{Y, K-1} & & \\ & & \ddots & \ddots & \\ \mathbf{0} & & & \ddots & \mathbf{D}^{Y, 2} \\ \mathbf{0} & & & & \mathbf{0}\end{array}\right)$, for $Y=C_{1}, C_{2}$ and

$\mathbf{D}^{Y}=\left(\begin{array}{cccc}\mathbf{0} & \cdots & \cdots & \mathbf{0} \\ \vdots & \ddots & & \vdots \\ \mathbf{0} & & \ddots & \vdots \\ \mathbf{D}^{Y, 1} & \mathbf{0} & \cdots & \mathbf{0}\end{array}\right)$

for $Y=F C_{1}, F C_{2}$.

Matrix $\mathbf{D}^{Y, k}$ for $Y=O, C_{1}, C_{2}, F C_{1}, F C_{2}$

The elements of the matrix $\mathbf{D}^{Y, k}$ for $k=1, \ldots, K$ and $Y=O, C_{1}, C_{2}$, $F C_{1}, F C_{2}$, are given by

$\mathbf{D}^{Y, k}= \begin{cases}\left(\mathbf{D}_{l h}^{Y, k}\right)_{l, h=0, \ldots, k} & ; \quad Y=O \\ \left(\mathbf{D}_{l h}^{Y, k}\right)_{\substack{l=0, \ldots, k \\ h=0, \ldots, k-1}} & ; \quad Y=C_{1}, C_{2} \\ \left(\mathbf{D}_{l h}^{Y, 1}\right)_{\substack{l=0,1 \\ h=0, \ldots, K}} & Y=F C_{1}, F C_{2} ; k=1\end{cases}$

where $\mathbf{D}_{l h}^{O, k}=\mathbf{0}$ if $h>l$ or $h<l-\min \left\{l, R_{k}\right\}, \mathbf{D}_{l h}^{C_{i}, k}=\mathbf{0}$ if $h>l$ or $h<l-\min \left\{l, R_{k}\right\}$ or $l=k$ and $\mathbf{D}_{l h}^{F C_{i}, 1}=\mathbf{0}$ for all $l$ and $h$ excepting for the case $l=h=0$.

For $k=1, \ldots, K$,

$\mathbf{D}_{00}^{Y, k}=\left\{\begin{array}{ll}\mathbf{H}_{0}+I_{\{k=1\}} \mathbf{H}_{\mathrm{mr}}^{\prime} ; & Y=O \\ \mathbf{H}_{\mathrm{nrep}}^{\text {type }} ; & Y=C_{\text {type }} \text { or } Y=F C_{\text {type }}\end{array}\right.$,

For $l=1, \ldots, R_{k}$

$\mathbf{D}_{l, 0}^{Y, k}\left(j_{1}, \ldots, j_{l}\right)= \begin{cases}\mathbf{H}_{0} \otimes E\left(k, l, l, 0 ; j_{1}, \ldots, j_{l} ; 0,0\right) & ; \quad Y=O \text { and } l<k \\ \mathbf{H}_{\text {nrep }}^{\text {yrep }} \otimes E\left(k, l, l, 0 ; j_{1}, \ldots, j_{l}, 0,1\right) & ; \quad Y=C_{\text {type }} \text { and } l<k, \\ \zeta \otimes E\left(k, l, l, 0 ; j_{1}, \ldots, j_{l} ; 0,0\right) & ; \quad Y=O \text { and } l=k\end{cases}$

with $\zeta=\boldsymbol{\alpha}$ for system $I, \zeta=\boldsymbol{\alpha} \otimes\left(\mathbf{L}+\mathbf{L}^{0} \boldsymbol{\gamma}\right)$ for system II and $\zeta=\boldsymbol{\alpha} \otimes$ $\left(\mathbf{L}+\mathbf{L}^{0} \boldsymbol{\gamma}\right) \otimes \boldsymbol{\eta} \otimes \boldsymbol{\omega}$ for system III.

For $l=1, \ldots, k-1 ; a=0, \ldots, \min \left\{R_{k}, l-1\right\}$ with $k>1$,

$\mathbf{D}_{l, l-a}^{Y, k}\left(i_{1}, \ldots, i_{l-a} ; j_{1}, \ldots, j_{l}\right)$

$=\left\{\begin{array}{l}{\left[\mathbf{H}_{0}+I_{\{l=k-1 \text { and } a=0\}} \mathbf{H}_{\text {mr }}^{\prime}\right] \otimes E\left(k, l, a, 0 ; i_{1}, \ldots, i_{l-a} ; j_{1}, \ldots, j_{l} ; 0,0\right) ; \quad Y=O} \\ \left(\mathbf{H}_{\text {nrep }}^{\text {type }} \boldsymbol{I}_{\{l<k-1 \text { lora } a>0\}}+\mathbf{H}_{\text {nrep }}^{\prime \text { type }} \boldsymbol{I}_{\{l=k-1 \text { and } a=0\}}\right) \\ \quad \otimes E\left(k, l, a, b ; i_{1}, \ldots, i_{l-a} ; j_{1}, \ldots, j_{l} ; 0,1\right) ; \quad Y=C_{t y p e}\end{array}\right.$.

For $\mathrm{a}=1, \ldots, \min \left\{R_{k}, k-1\right\}$,

$\mathbf{D}_{k, k-a}^{O, k}\left(i_{1}, \ldots, i_{k-a} ; j_{1}, \ldots, j_{k}\right)=\zeta \otimes E\left(k, k, a, 0 ; i_{1}, \ldots, i_{k-a} ; j_{1}, \ldots, j_{k} ; 0,0\right)$, with $k>1$.

For $k=1, \ldots, K$,

$\mathbf{D}_{k, k}^{O, k}\left(i_{1}, \ldots, i_{k} ; j_{1}, \ldots, j_{k}\right)$

$=\left\{\begin{array}{l}E\left(k, k, 0,0 ; i_{1}, \ldots, i_{k} ; j_{1}, \ldots, j_{k} ; 0,0\right) ; \\ \left(\mathbf{L}+\mathbf{L}^{0} \boldsymbol{\gamma}\right) \otimes E\left(k, k, 0,0 ; i_{1}, \ldots, i_{k} ; j_{1}, \ldots\right.\end{array}\right.$

system $I$

$=\left\{\left(\mathbf{L}+\mathbf{L}^{0} \boldsymbol{\gamma}\right) \otimes E\left(k, k, 0,0 ; i_{1}, \ldots, i_{k} ; j_{1}, \ldots, j_{k} ; 0,0\right) ; \quad\right.$ systems $I I, I I I$

Matrix $\mathbf{D}^{A_{i}, k}$

The elements of the matrix $\mathbf{D}^{A_{i}, k}$ for $i=1,2$ and fork $=1, \ldots, K$ are given by

$\mathbf{D}^{A_{i}, k}=\left(\mathbf{D}_{l h}^{A_{i}, k}\right)_{l, h=0, \ldots, k}$

where $\mathbf{D}_{l h}^{A_{i}, k}=\mathbf{0}$ if $h>l+1$ or $h<l+1-\min \{l, R(k)\}$ or $l=k$.
For type $=1,2$, then

$\mathbf{D}_{01}^{A_{t y p e}, k}(1)=\left(\mathbf{H}_{\text {rep }}^{\text {type }} I_{\{k>1\}}+\mathbf{H}_{\text {rep }}^{\prime \text { type }} I_{\{k=1\}}\right) \otimes E(k, 0,0,0 ; 1 ; 1,0)$.

For $l=1, \ldots, k-1 ; a=0, \ldots, \min \left\{l, R_{k}\right\}$ with $k>1$

$$
\begin{aligned}
& \mathbf{D}_{l, l+1-a}^{A_{\text {type }}, k}\left(i_{1}, \ldots, i_{l+1-a-1}, 1 ; j_{1}, \ldots, j_{l}\right) \\
& \quad=\left(\mathbf{H}_{\text {rep }}^{\text {type }} I_{\{l<k-1 \text { or } a>0\}}+\mathbf{H}_{\text {rep }}^{\prime \text { type }} I_{\{l=k-1 \text { and } a=0\}}\right) \\
& \quad \otimes E\left(k, l, a, 0 ; i_{1}, \ldots, i_{l+1-a-1}, 1 ; j_{1}, \ldots, j_{l} ; 1,0\right)
\end{aligned}
$$

Matrix $\mathbf{D}^{B_{i}, k}$

The elements of the matrix $\mathbf{D}^{B_{i}, k}$ for $i=1,2,3$ and for $k=1, \ldots, K$ are given by

$\mathbf{D}^{B_{i}, k}=\left(\mathbf{D}_{l h}^{B_{i}, k}\right)_{l, h=0, \ldots, k}$

where $\mathbf{D}_{l h}^{A_{i}, k}=\mathbf{0}$ if $h>l+1$ or $h<l+1-\min \left\{l, R_{k}\right\}$ or $l \geq k-1$.

For type $=1,2,3$ then

$\mathbf{D}_{01}^{B_{t y p e}, k}(2)=\mathbf{H}_{\mathrm{mr}}^{\text {type }} I_{\{k>1\}} \otimes E(k, 0,0,0 ; 2 ; 1,0)$.

For $l=1, \ldots, k-2 ; a=0, \ldots, \min \left\{l, R_{k}\right\}$ with $k>1$

$$
\begin{aligned}
& \mathbf{D}_{l, l+1-a}^{B_{t y p e}, k}\left(i_{1}, \ldots, i_{l+1-a-1}, 2 ; j_{1}, \ldots, j_{l}\right) \\
& \quad=\mathbf{H}_{\mathrm{mr}}^{t y p e} \otimes E\left(k, l, a, 0 ; i_{1}, \ldots, i_{l+1-a-1}, 2 ; j_{1}, \ldots, j_{l} ; 1,0\right)
\end{aligned}
$$

\section{References}

[1] Artalejo JR, Gómez-Corral A, He QM. Markovian arrivals in stochastic modelling: a survey and some new results. Stat Oper Res Trans 2010;34(2):101-144.

[2] Barbu VS, Limnios N. Reliability of semi-Markov systems in discrete time: modeling and estimation. Handbook of performability engineering. Springer-Verlag London Limited; 2008

[3] Buchholz P, Kriege J, Felko I. Input modeling with phase-type distributions and Markov models. Theory and applications. Heidelberg New York Dordrecht London: Springer Cham; 2014.

[4] Daneshkhah A, Stocks NG, Jeffrey P. Probabilistic sensitivity analysis of optimised preventive maintenance strategies for deteriorating infrastructure assets. Reliab Eng Syst Saf 2017;163:33-45.

[5] Das S, Dewanji A, Segupta D. Discrete time software reliability modeling with periodic debugging schedule. Stat Method 2016;33:147-159.

[6] Dewanji A, Segupta D, Chakraborty K. A discrete time model for software reliability with application to a flight control software. Appl Stochastic Models Bus Ind 2011;27(6):723-731.

[7] He Q-M. Fundamentals of matrix-analytic methods. New York Heidelberg Dordrecht London: Springer; 2014.

[8] Georgiadis S, Limnios N. Interval reliability for semi-Markov systems in discrete time. J de la Société Française de Statistique 2014;153(3):152-166.

[9] Kim H, Kim P. Reliability-redundancy allocation problem considering optimal redundancy strategy using parallel genetic algorithm. Reliab Eng Syst Saf 2017:159:153-160.

[10] Levitin G, Finkelstein M, Dai Y. Redundancy optimization for series-parallel phased mission systems exposed to random shocks. Reliab Eng Syst Saf 2017;167:554-560.

[11] Levitin G, Jia H, Ding Y, Song Y, Dai Y. Reliability of multi-state systems with free access to repairable standby elements. Reliab Eng Syst Saf 2017;167:192-197.

[12] Levitin G, Xing L, Dai Y. Cold vs. hot standby mission operation cost minimization for 1-out-of-N systems. Eur J Oper Res 2014;234:155-162.

[13] Li Y, Cui L, Lin C. Modeling and analysis for multi-state systems with discrete-time Markov regime-switching. Reliab Eng Syst Saf 2017;166:41-9.

[14] Lisnianski A, Frenkel I, Khvatskin L. On sensitivity analysis of aging multi-state system by using LZ-transform. Reliab Eng Syst Saf 2017;166:99-108.

[15] Markopoulos T, Platis AN. Reliability analysis of a modified IEEE 6BUS RBTS multi-state system. In: Recent advances in multi-state systems reliability. Springer; 2017. p. 301-19.

[16] Murchland J. Fundamental concepts and relations for reliability analysis of multistate systems. In: Barlow RE, Fussell JB, Singpurwalla N, editors. Reliability and fault tree analysis: theoretical ans applied aspects of system reliability. Philadelphia: SIAM; 1975. p. 591-618

[17] Nakagawa T. Maintenance theory on reliability. Springer-Verlag London Limited; 2005 .

[18] Neuts MF. A versatile Markovian point process. J Appl Probab 1979;16:764-79.

[19] Neuts MF. Matrix geometric solutions in stochastic models. an algorithmic approach. Dover Publications; 1981.

[20] Neuts MF. probability distributions of phase type. In: Florin Emeritus H, editor. Liber amicorum. Belgium: Department of Mathematics, University of Louvain; 1975. p. $173-206$.

[21] Okamura H, Dohi T, Trivedi KS. Markovian arrival process parameter estimation with group data. IEEE/ACM Trans Networking 2009;17(4):1326-1339. 
[22] Peng R, Xiao H, Liu H. Reliability of multi-state systems with a performance sharing group of limited size. Reliab Eng Syst Saf 2017;166:164-170.

[23] Qiu Q, Cui L, Gao H. Availability and maintenance modelling for systems subject to multiple failure modes. Comput Ind Eng 2017;108:192-198.

[24] Ruiz-Castro JE. A preventive maintenance policy for a standby system subject to internal failures and external shocks with loss of units. Int J Syst Sci 2015;46(9): $1600-1613$.

[25] Ruiz-Castro JE. Complex multi-state systems modelled through marked Markovian arrival processes. Eur J Oper Res 2016;252(3):852-65.

[26] Ruiz-Castro JE, Fernández-Villodre G. A complex discrete warm standby system with loss of units. Eur J Oper Res 2012;218:456-69.

[27] Ruiz Castro JE, Li QL. Algorithm for a general discrete $k$-out-of- $n$ : $G$ system subject to several types of failure with an indefinite number of repairpersons. Eur J Oper Res 2011;211(1):97-111.

[28] Shatnawi O. An integrated framework for developing discrete-time modelling in software reliability engineering. Qual Reliab Eng Int 2016;32(8):2925-2943.
[29] Warrington L, Jones JA. Representing complex systems within discrete event simulation for reliability assessment. In: Proceedings annual reliability and maintainability symposium; 2003. p. 487-92.

[30] Wells CE. Reliability analysis of a single warm-standby system subject to repairable and nonrepairable failures. Eur J Oper Res 2014;235(1):180-186.

[31] Xie Y, Zhang J, Aldemir T, Denning R. Multi-state Markov modeling of pitting corrosion in stainless steel exposed to chloride-containing environment. Reliab Eng Syst Saf 2018;172:239-248

[32] Yi H, Cui L. Distribution and availability for aggregated second-order semi- Markov ternary system with working time omission. Reliab Eng Syst Saf 2017;166:50-60.

[33] Yeh C-T, Fiondella L. Optimal redundancy allocation to maximize multi-state computer network reliability subject to correlated failures. Reliab Eng Syst Sa 2017;166:138-150.

[34] Yongjin Z, Youchao S, Longbiao L, Ming Z. Copula-based reliability analysis for a parallel system with a cold standby. Commun Stat 2018;47(3):562-582. 